\title{
Swivel-Cruciform Stilbenes Based on Bithiophene
}

\author{
Amaranda García-Rodríguez, ${ }^{[\mathrm{a}]}$ Antonio M. Rodríguez, ${ }^{[\mathrm{a}]}$ Pilar Prieto, ${ }^{[\mathrm{a}]}$ Raquel Andreu, ${ }^{[\mathrm{b}]}$ \\ Sonia Merino, ${ }^{[a]}$ and Julián Rodríguez-López ${ }^{\star[a]}$
}

\begin{abstract}
Bithiophene-based cruciforms with different stilbenoid arms at the 3,3'- and 5,5'-positions have been synthesized by various combinations of Suzuki and Horner-Wadsworth-Emmons (HWE) reactions. According to DFT calculations, the steric hindrance between the arms at the 3,3'-positions produces a twist angle of $57.6^{\circ}$ between the two thiophene rings that form the 2,2'-bithiophene unit, an arrangement that leads to a swivel-cruciform structure. The UV-vis spectra contained strong absorption bands at wavelengths consistent with a twisted molecule with little interaction between the arms. The ability of these compounds to form highly stable radical cations was demonstrated by cyclic voltammetry and this, together with their good solubility in organic solvents, indicates that these materials have potential for the development of solution-processed electronic devices.
\end{abstract}

\section{Introduction}

Conjugated organic compounds are recognized as ideal materials in device applications. ${ }^{[1]}$ Among them, functionalized cruciform-shaped $\pi$-conjugated compounds have attracted significant interest due to their excellent optoelectronic properties. These properties arise because of the unique, multiply conjugated pathway structures, which consist of two distinct molecular axes with either the same or different $\pi$ conjugated arms. ${ }^{[2]}$ This type of compound exhibits the common feature of having the highest occupied molecular orbital (HOMO) and the lowest unoccupied molecular orbital (LUMO) geometrically separated. As a consequence, the attachment of stimuli-responsive functionalities on the axes means that the energy of one frontier orbital may be perturbed while that of the other remains fairly constant. Researchers have taken advantage of this property to use such materials in supramolecular assemblies, ${ }^{[3]}$ as switches in molecular electronics, ${ }^{[4]}$ in organic field-effect transistors (OFETs), ${ }^{[5]}$ for electroluminescent devices, ${ }^{[6]}$ dye-sensitized solar cells, ${ }^{[7]}$ and, most notably, as responsive cores in sensory schemes. ${ }^{[8]}$ In recent years, swivel-cruciforms have received increasing

[a] Dr. A. García-Rodríguez, Dr. A. M. Rodríguez, Prof.. P. Prieto, Prof. S. Merino, Prof. J. Rodríguez-López

Área de Química Orgánica. Facultad de Ciencias y Tecnologías Químicas. Universidad de Castilla-La Mancha

Avda. Camilo José Cela 10, 13071 Ciudad Real (Spain)

E-mail: sonia.merino@uclm.es; julian.rodriguez@uclm.es

[b] Prof. R. Andreu

Departamento de Química Orgánica-ICMA

Universidad de Zaragoza-CSIC,

Pedro Cerbuna 12, 50009 Zaragoza (Spain)

Supporting information for this article is available on the WWW under http://dx.doi.org/10.1002/ejoc.201xxxxxx. attention, in particular those based on thiophene compounds, which are considered to be a new class of semiconducting materials with excellent solubility properties and good suitability for organic devices. ${ }^{[9]}$ In biothiophene-based derivatives, the term swivel-cruciform refers to the fact that the single bond between the two thiophene rings allows rotation between the arms and provides the possibility of changing the value of the dihedral angle between the two rings, thus modulating the electron delocalization along the main chain axis. Additionally, this situation leads to weaker intermolecular interactions and increased solubility.

In the search for new soluble structures with a tunable band gap and, therefore, tunable optoelectronic properties, we report here the synthesis and full characterization of new bithiophenecentered swivel cruciform derivatives that contain stilbene arms in the 3,3'- and 5,5'-positions directions. The arms bear either identical or different end-substituents with a variety of donorand/or acceptor groups (Chart 1). The geometries and electronic structures of the compounds were analyzed theoretically at the $a b$ initio density functional level. In addition, the photophysical and electrochemical properties of these systems are also reported.

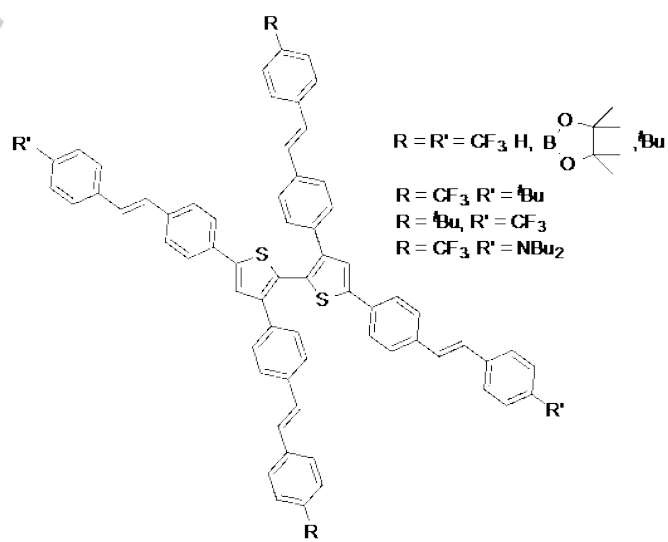

Chart 1. Bithiophene-centered Swivel Cruciforms.

\section{Results and Discussion}

Different methodologies were used for the synthesis of the bithiophene-based cruciforms. The approaches all involved a combination of Suzuki and Horner-Wadsworth-Emmons (HWE) reactions. The compounds with different end substituents were prepared from dialdehyde $\mathbf{2}$, which is easily accessible by Suzuki coupling between 3,3'-dibromo-2,2'-bithiophene and 4formylphenylboronic acid followed by bromination of the 5,5'positions with NBS (Scheme 1). 


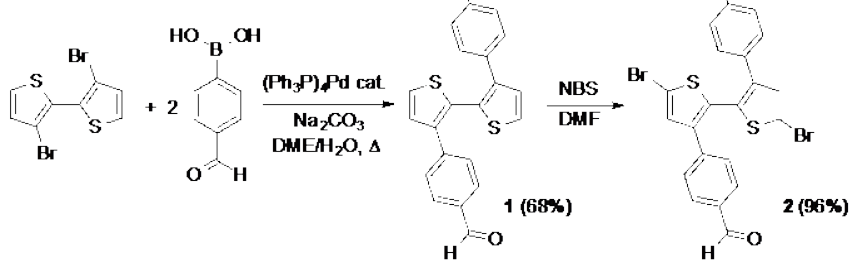

Scheme 1. Synthesis of Dialdehyde 2

Subsequent HWE reaction of $\mathbf{2}$ with the corresponding parasubstituted benzylphosphonate led to compounds $\mathbf{3} \mathbf{a}-\mathbf{b}$, which after a new Suzuki coupling with 4-formylphenylboronic acid gave compounds $\mathbf{4} \mathbf{a}-\mathbf{b}$ in good yields. Finally, a second HWE reaction with the appropriate benzylphosphonate produced cruciforms $\mathbf{5 a} \mathbf{a}-\mathbf{b}$ with different end substituents (Scheme 2). coupling with 3a under microwave assistance (Scheme 3). This modified protocol led to the successful synthesis of compound $\mathbf{5 c}$ in good yield and with high purity.

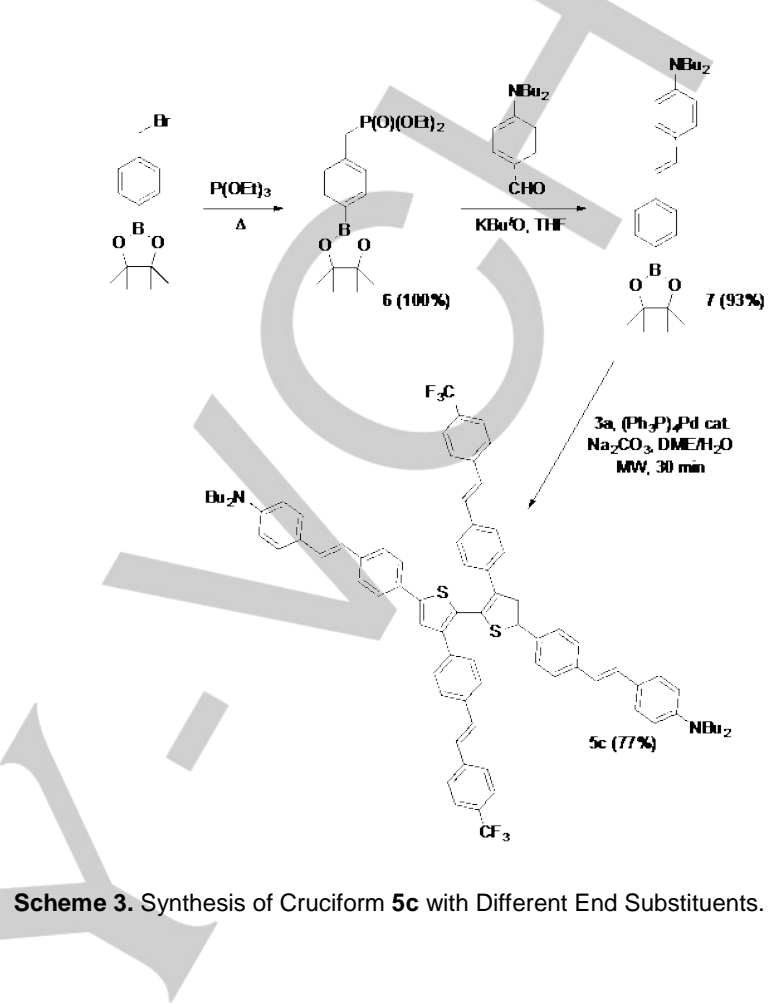

3,3',5,5'-Tetrabromo-2,2'-bithiophene was used as the starting material for the synthesis of cruciforms with the same end substituents. In this case, treatment with 4-formylphenylboronic acid readily gave tetraaldehyde $\mathbf{8}$, which could be reacted with a variety of para-substituted benzylphosphonates to give the target products $\mathbf{9 a - c}$ in good yields (Scheme 4).
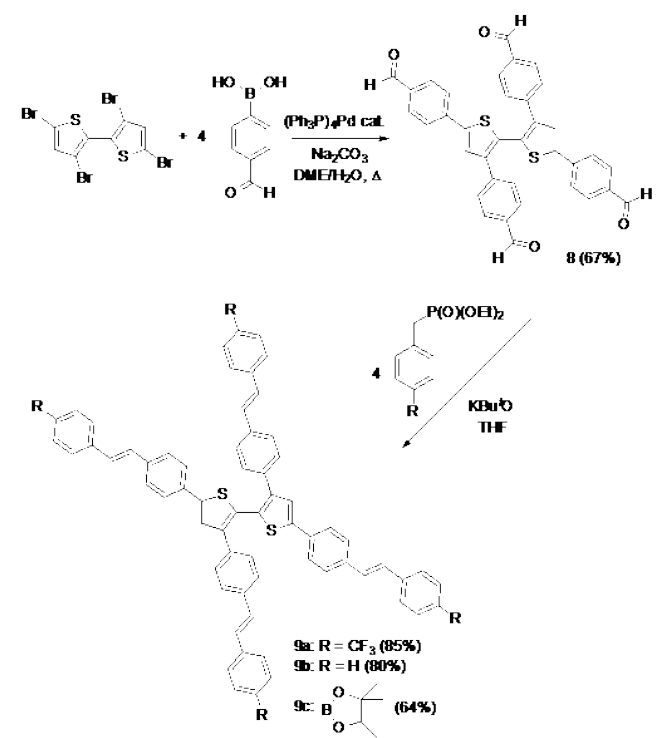

Scheme 4. Synthesis of Bithiophene-Based Cruciforms 9a-c with Identical End Substituents. 
Once again, the HWE reactions could not be performed with electron-rich benzyl phosphonates. The direct reaction with four equivalents of para-tert-butylbenzyl phosphonate was unsuccessful and the corresponding cruciform $9 \mathrm{~d}$ could only be prepared from dialdehyde $\mathbf{4 b}$ in moderate yield (Scheme 5).

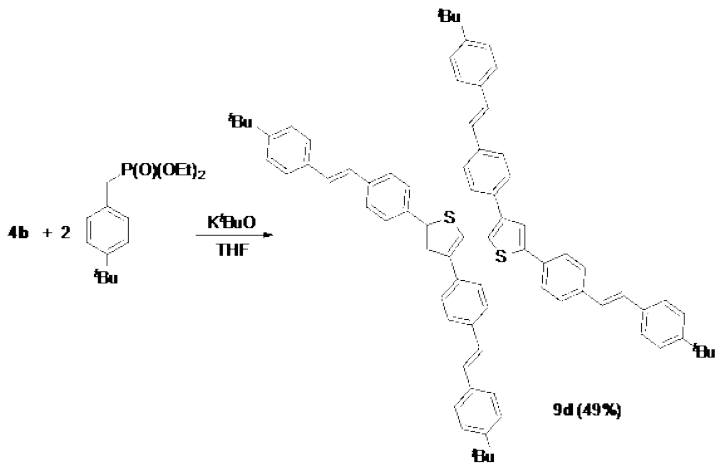

Scheme 5. Synthesis of Cruciform 9d with Identical End Substituents.

DFT calculations were performed on compounds $\mathbf{5}$ and $\mathbf{9}$ at the B3LYP/6-31G* level of theory. The optimized gas phase structure for cruciform $\mathbf{9 b}$, as a model for the series, is depicted in Figure 1 (see Supporting Information for all cruciforms). As can be seen, the molecule adopts a swivel configuration with four stilbene arms pointing in different directions to produce a distorted tetrahedral geometry. The torsion of the centra bithiophene unit, in which the two thiophene rings adopt a transorientation, is generated by the steric hindrance between the stilbene arms attached at the 3,3'-positions, with a twist angle of $57.6^{\circ}$ between the two thiophene mean planes. Furthermore, the benzene rings at the $3,3^{\prime}$ - and 5,5'-positions are bound to the bithiophene with twist angles of $38.8^{\circ}$ and $24.0^{\circ}$, respectively. Other authors have found comparable values in related terthiophenes. ${ }^{[11]}$
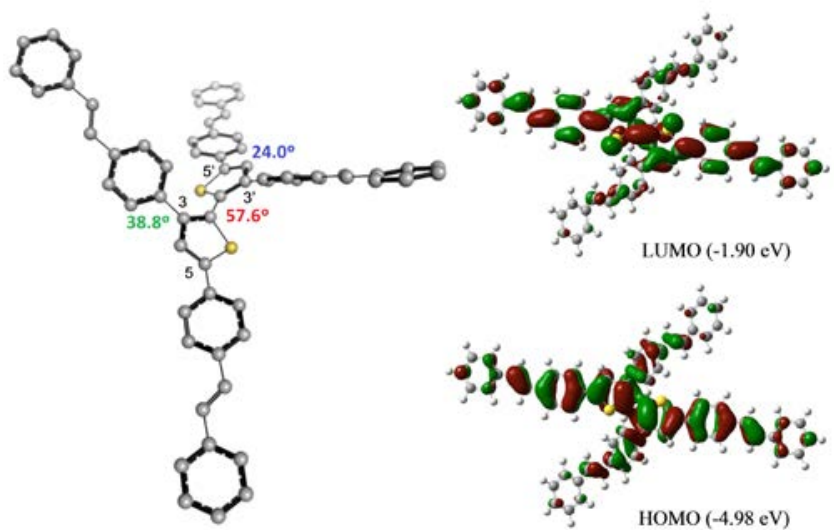

Figure 1. Optimized structure and frontier orbitals of $9 b$

The calculated frontier orbitals for compound $\mathbf{9 b}$, which has four identical end substituents, are also shown in Figure 1 (see Supporting Information for $\mathbf{9 a , c - d}$ ). Both the HOMO and LUMO are overlapped and almost extend over the entire molecule, mainly over the bithiophene unit and the arms in the 5,5' positions - a situation in agreement with the higher planarity observed in this part of the structure (see above). Thus, the twisted central bithiophene does not fully interrupt electron delocalization between the conjugated arms. The gas phase HOMO-LUMO energy gap was also calculated for $\mathbf{9 b}$ and $a$ value of $3.08 \mathrm{eV}$ was obtained.

A different situation was found for compounds $\mathbf{5}$, which have different end substituents, especially in the case of compound $\mathbf{5 c}$ with strong electron-donor groups $\left(\mathrm{NBu}_{2}\right)$. In this case (Figure 2), the HOMO is essentially localized over the arms bearing the donor groups, whereas the LUMO is mostly localized over the arms with the electron-acceptor groups $\left(\mathrm{CF}_{3}\right)$ (see Supporting Information for $\mathbf{5 a - b}$ ). The bithiophene unit incorporates both frontier orbitals and the HOMO-LUMO energy gap was reduced to $2.74 \mathrm{eV}$, mainly due to the increase in the HOMO energy level caused by the presence of strong donor groups.

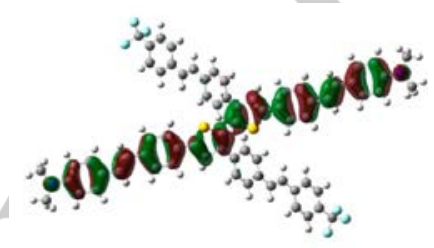

HOMO $(-4.67 \mathrm{eV})$

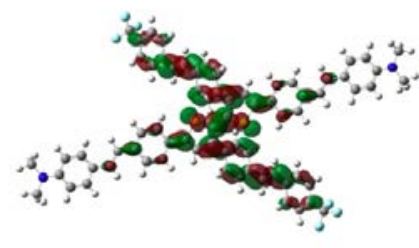

LUMO (-1.93 eV)
Figure 2. Frontier orbitals of $\mathbf{5 c}$. The $\mathrm{NBu}_{2}$ groups have been replaced by $\mathrm{NMe}_{2}$ groups to save computational time.

As one would expect, the swivel-cruciform structure of the molecules endow them with increased solubility in common organic solvents, a characteristic that is further improved when terminal alkyl substituents are attached. This good solubility allowed a detailed characterization by NMR spectroscopy. The ${ }^{3} \mathrm{~J}(\mathrm{H}, \mathrm{H})$ coupling constants of $\sim 16 \mathrm{~Hz}$ for the vinylic protons in the ${ }^{1} \mathrm{H}$ NMR spectra clearly support the selective formation of $E$ configured double bonds in all cases. In addition to the vinylic signals, the spectra showed several differentiated $A B$ systems with ${ }^{3} \mathrm{~J}(\mathrm{H}, \mathrm{H}) \sim 8 \mathrm{~Hz}$ in the aromatic region, assigned to the different para-disubstituted benzene rings (Figure 3). The MALDI-TOF mass spectrometry data also proved to be important for the identification of the compounds.

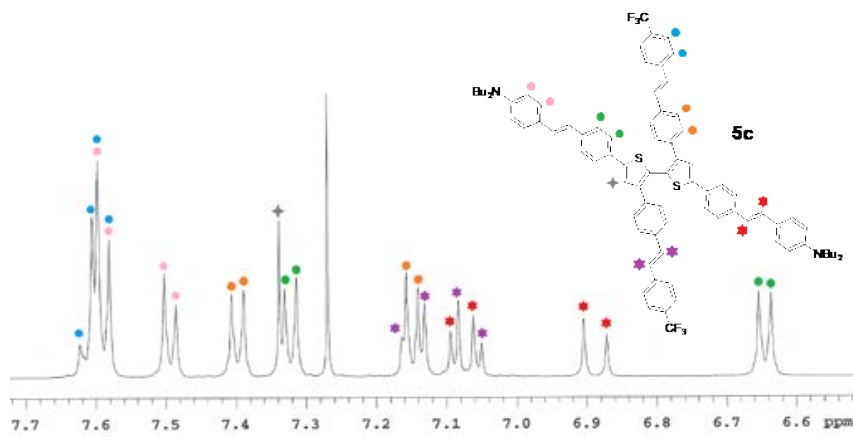

Figure 3. Aromatic region of the ${ }^{1} \mathrm{H}$ NMR spectrum of $5 \mathrm{c}\left(500 \mathrm{MHz}, \mathrm{CDCl}_{3}\right)$ Two $A B$ quartets can be observed for the double bonds with $\mathrm{J} \sim 16 \mathrm{~Hz}$ (red and purple stars), together with four extra $A B$ quartets with $J \sim 8 \mathrm{~Hz}$ assigned to the different benzene rings (colored circles). 
It is a prerequisite to study the photophysical and electrochemical properties of materials when considering applications in organic devices. The photophysical properties of the synthesized cruciforms were examined by UV-vis and fluorescence spectroscopy in DCM at $25{ }^{\circ} \mathrm{C}$. The results are listed in Table 1. With the exception of compound $\mathbf{5 c}$, which has different end substituents, all of the absorption spectra had a similar shape, namely a $\pi-\pi^{\star}$ transition at $\lambda_{\max }=341-347 \mathrm{~nm}$ with large absorption coefficient $(\varepsilon)$ due to the stilbene arms (Figure 4, see also Supporting Information). The spectrum of compound 5c showed two maxima at 331 and $397 \mathrm{~nm}$ (Figure 4), where the second maximum can be assigned to the arms bearing the dibutylamino group. The UV-vis data are consistent with the twist of the molecule and imply that there is little electronic interaction between the arms in the ground state. With respect to the fluorescence features, small bands with vibronic structure were observed in the range 356-427 nm together with a large band at $527-530 \mathrm{~nm}$. The latter band is significantly redshifted for $5 \mathrm{c}\left(\right.$ ca. $35 \mathrm{~nm}, \lambda_{\max }=564 \mathrm{~nm}$ ), which is consistent with the lower HOMO-LUMO energy gap calculated for this compound (see above).

Table 1. Optical Spectroscopy Data for Cruciforms $\mathbf{5}$ and $\mathbf{9}$

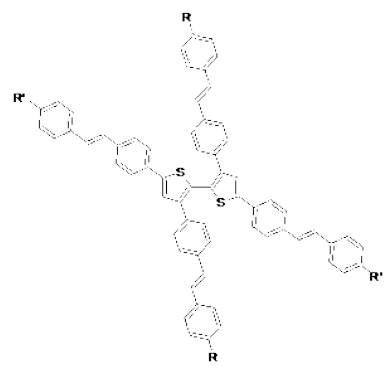

\begin{tabular}{|c|c|c|c|c|}
\hline Compd $^{[a]}$ & $\mathrm{R}$ & $\mathrm{R}^{\prime}$ & $\lambda_{\mathrm{abs}}, \mathrm{nm}\left(\varepsilon, \mathrm{mM}^{-1} \cdot \mathrm{cm}^{-1}\right)$ & $\lambda_{\mathrm{em}}, \mathrm{nm}$ \\
\hline $5 a$ & $\mathrm{CF}_{3}$ & ${ }^{t} \mathrm{Bu}$ & $345(97.1)$ & $405,427,530$ \\
\hline $5 b$ & ${ }^{t} \mathrm{Bu}$ & $\mathrm{CF}_{3}$ & $344(150.5)$ & $408,427,528$ \\
\hline $5 c$ & $\mathrm{CF}_{3}$ & $\mathrm{NBu}_{2}$ & 331 (97.5), 397 (102.7) & $409,426,564^{[b]}$ \\
\hline $9 a$ & $\mathrm{CF}_{3}$ & $\mathrm{CF}_{3}$ & $344(115.6)$ & $406,426,529$ \\
\hline $9 b$ & $\mathrm{H}$ & $\mathrm{H}$ & $343(129.2)$ & $401,422,529$ \\
\hline 9c & & & $341(50.6)$ & $356,374,393,527$ \\
\hline 9d & ${ }^{t} \mathrm{Bu}$ & ${ }^{t} \mathrm{Bu}$ & $347(150.4)$ & $406,427,529$ \\
\hline
\end{tabular}

[a] All spectra were recorded in DCM solutions at room temperature at $c$ $=1.00-5.23 \times 10^{-6} \mathrm{M}$. [b] Excitation at $331 \mathrm{~nm}$.
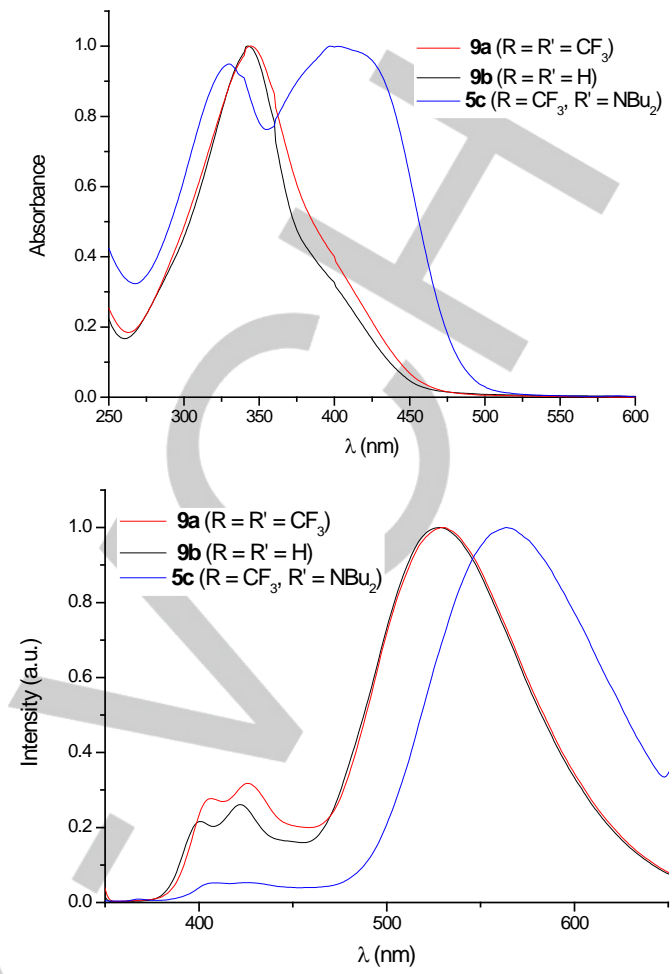

Figure 4. Normalized absorption and emission spectra of cruciforms $\mathbf{9 a -} \mathbf{b}$ and $5 c$ in DCM. See Supporting Information for data for all compounds.

It is worth noting that the fluorescence spectra of $\mathbf{5 c}$ showed significant solvatochromism (Table 2, Figure 5), a finding that is indicative of a highly polarized singlet excited state generated by the intramolecular charge transfer from the arms bearing the $\mathrm{NBu}_{2}$ groups to those bearing the $\mathrm{CF}_{3}$ groups. This situation also leads to a moderate quantum yield, with significant differences not observed between solvents. Nevertheless, solvatochromism is almost negligible in the absorption spectra and this finding supports the absence of a significant electronic interaction between the arms in the ground state.

Table 2. UV/Vis and Fluorescence data for $5 c\left(R=\mathrm{CF}_{3}, \mathrm{R}^{\prime}=\mathrm{NBu}_{2}\right)$ in different solvents.

\begin{tabular}{llll}
\hline Solvent & $\lambda_{\mathrm{abs}}, \mathrm{nm}$ & $\lambda_{\mathrm{em}}, \mathrm{nm}^{[\mathrm{a}]}$ & $\Phi_{\mathrm{F}}{ }^{[\mathrm{b}]}$ \\
\hline Hexane & 327,394 & 529 & 0.08 \\
DCM & 331,397 & 564 & 0,07 \\
MeOH & 330,396 & 557 & 0,06 \\
Acetonitrile & 329,397 & 609 & 0,10 \\
DMSO & 331,401 & 614 & 0,06 \\
\hline
\end{tabular}

[a] Excitation at the less energetic absorption band. [b] Fluorescence quantum yield $( \pm 10 \%)$ determined relative to quinine sulfate in $0.1 \mathrm{M}$ $\mathrm{H}_{2} \mathrm{SO}_{4}\left(\Phi_{\mathrm{F}}=0.54\right)$ and 9,10-diphenylanthracene in cyclohexane $\left(\Phi_{\mathrm{F}}=\right.$ $0.90)$ as standards. 


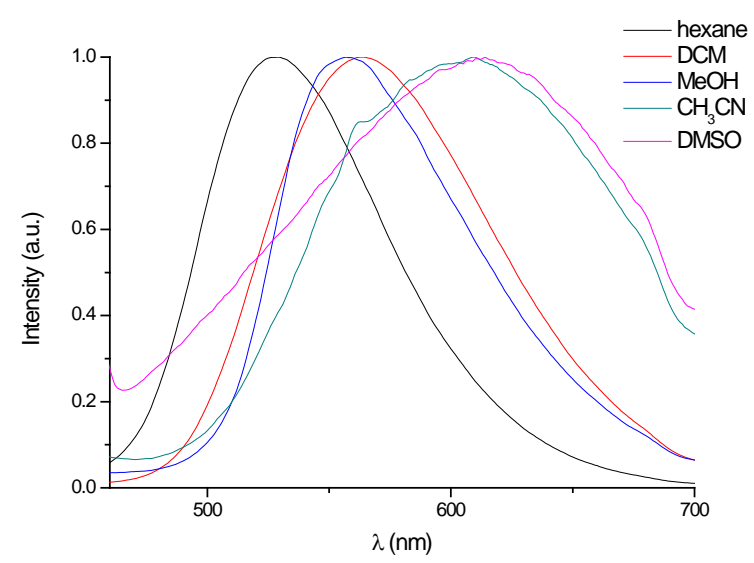

Figure 5. Normalized emission spectra of compound $\mathbf{5 c}$ in different solvents

The electrochemical properties of some compounds were analyzed in DCM at room temperature by cyclic voltammetry using $\mathrm{Bu}_{4} \mathrm{NPF}_{6}$ as the supporting electrolyte (Table 3, Figure 6). Two successive oxidation processes (three for compound $\mathbf{5 b}$ ) were detected for all analyzed compounds. With the exception of compound $\mathbf{5 c}$, the waves are reversible and this indicates the formation of a highly stable radical cation and a dication. This result demonstrates the potential of these cruciforms for holetransport in electronic devices. In the case of compound $\mathbf{5 b}$, the first two waves may correspond to two different radical cations stabilized on different conjugated arms, while the last wave could be assigned to a di-cation probably formed on the more planar part of the molecule with the more efficient conjugation length. ${ }^{\left[{ }^{[g]}\right.}$ As one would expect, the electron-acceptor character of the four $\mathrm{CF}_{3}$ groups means that compound $9 \mathrm{a}$ has higher potentials and these decrease by $0.05 \mathrm{~V}$ when substituents are not present $\left(9 b, R=R^{\prime}=H\right)$. The potentials of donor-acceptor systems $\mathbf{5 b}$ and $\mathbf{5 c}$ are lower than those obtained for $\mathbf{9 a}$. The decrease is very small for $\mathbf{5 b}$ but is more marked for compound $5 c$, which contains electron-donating $\mathrm{NBu}_{2}$ groups. Reduction processes were not observed between 0 and $-2 \mathrm{~V}$ in any case. The HOMO and LUMO energy levels referred to the vacuum level were estimated by combining electrochemical and optical data. The values obtained are summarized in Table 3 . The HOMO was determined from the oxidation potential by the empirical relationship: $\mathrm{HOMO}=-\left(E^{\mathrm{ox}}{ }_{\text {onset }}+4.4\right) \mathrm{eV}$ where $E^{\mathrm{ox}}{ }_{\text {onset }}$ is the onset potential for the first oxidation wave relative to the $\mathrm{Ag} / \mathrm{AgCl}$ reference electrode. ${ }^{[12]}$ The LUMO was deduced from the optical band gap using the expression: LUMO = HOMO + $\Delta E_{\text {gap }}$. The optical band gap, $\Delta E_{\text {gap }}$, considered as the HOMOLUMO separation, was estimated from the onset wavelength of the low absorption band. The qualitative trends derived from the experimental data are consistent with the results of DFT calculations, although some quantitative discrepancies were found due to solvent effects and intermolecular interactions are not considered in the theoretical calculations.
Table 3. Cyclic voltammetry data ${ }^{[a]}$ and energy level analysis for compounds $\mathbf{5 b}-\mathrm{c}$ and $\mathbf{9 a - b}$

\begin{tabular}{lllllll}
\hline Compd & $\begin{array}{l}E^{1}{ }_{1 / 2} \\
(\mathrm{~V})\end{array}$ & $\begin{array}{l}E^{2}{ }_{1 / 2} \\
(\mathrm{~V})\end{array}$ & $\begin{array}{l}E_{\text {onset }}^{1} \\
(\mathrm{~V})\end{array}$ & $\begin{array}{l}E_{\mathrm{HOMO}} \\
(\mathrm{eV})\end{array}$ & $\begin{array}{l}\Delta E_{\text {gap }} \\
(\mathrm{eV})\end{array}$ & $\begin{array}{l}E_{\text {LUMO }} \\
(\mathrm{eV})\end{array}$ \\
\hline $\mathbf{5 b}\left(\mathrm{R}={ }^{t} \mathrm{Bu}, \mathrm{R}^{\prime}=\mathrm{CF}_{3}\right)^{[\mathrm{c}]}$ & 1.07 & 1.30 & 0.97 & -5.37 & 2.70 & -2.67 \\
$\mathbf{5 c}\left(\mathrm{R}=\mathrm{CF}_{3}, \mathrm{R}^{\prime}=\mathrm{NBu}_{2}\right)$ & $0.63^{[\mathrm{d}]}$ & $1.25^{[\mathrm{d}]}$ & 0.52 & -4.92 & 2.57 & -2.35 \\
$\mathbf{9 a}\left(\mathrm{R}=\mathrm{R}^{\prime}=\mathrm{CF}_{3}\right)$ & 1.09 & 1.31 & 1.00 & -5.40 & 2.71 & -2.69 \\
$\mathbf{9 b}\left(\mathrm{R}=\mathrm{R}^{\prime}=\mathrm{H}\right)$ & 1.04 & 1.26 & 0.93 & -5.33 & 2.73 & -2.60 \\
\hline
\end{tabular}

[a] 1.03-5.00 $\times 10^{-4} \mathrm{M}$ in $\mathrm{DCM}$ versus $\mathrm{Ag} / \mathrm{AgCl}(3 \mathrm{M} \mathrm{KCl})$, glassy carbon working electrode, Pt counter electrode, $20^{\circ} \mathrm{C}, 0.1 \mathrm{M} \mathrm{NBu}_{4} \mathrm{PF}_{6}, 100 \mathrm{mV} \mathrm{s}^{-1}$ scan rate. Ferrocene internal reference $E^{1 / 2}=+0.46 \mathrm{~V}$. $E_{1 / 2}^{1}$ : corresponds to the first oxidation couple and $E_{1 / 2}^{2}$ : corresponds to the second oxidation couple. [b] Onset potential for the first oxidation wave. [c] There is a third oxidation process at $1.65 \mathrm{~V}$. [d] Irreversible process: $E_{\text {ox }}$ value.
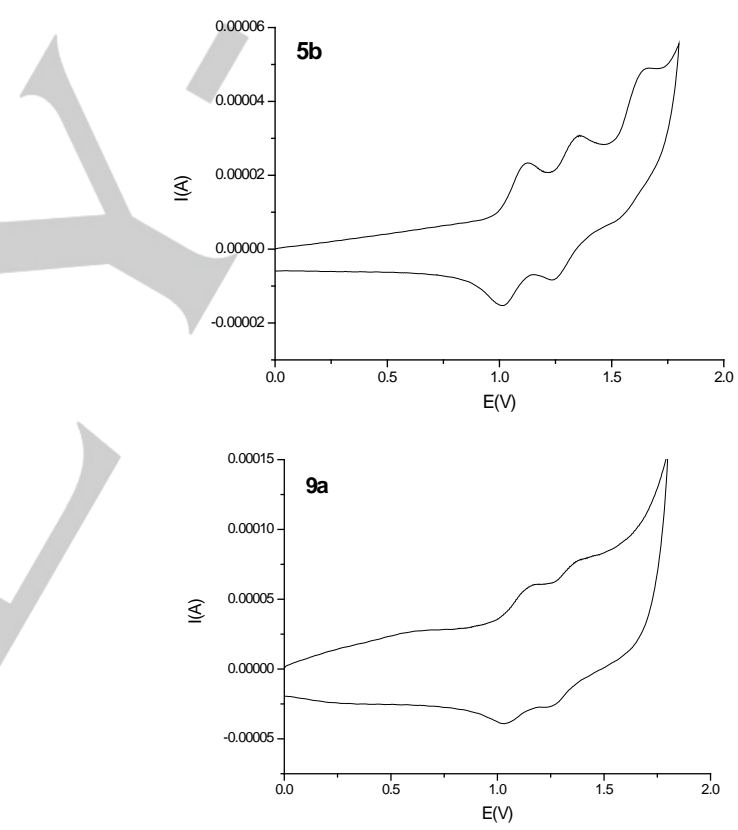

Figure 6. Cyclic voltammograms of compounds $\mathbf{5 b}\left(c=5.00 \times 10^{-4} \mathrm{M}\right)$ and $\mathbf{9 a}$ $\left(c=4.08 \times 10^{-4} \mathrm{M}\right)$. See Supporting Information for data for $5 c$ and $9 b$.

\section{Conclusions}

In summary, efficient synthetic routes that combine Suzuki and HWE reactions have been developed for the preparation of a new family of bithiophene-based cruciforms with stilbene arms at the 3,3'- and 5,5'-positions. The arms contain either the same or different end-substituents with diverse donor and/or acceptor groups. The steric hindrance between the stilbene arms at the 3,3 '-positions makes the molecules adopt a twisted geometry 
that results in weaker intermolecular interactions and better solubility in common organic solvents. All of the compounds present absorption wavelengths that confirm insignificant electronic interaction between the arms in the ground state. In compound $\mathbf{5 c}$ the introduction of strong electron-donating dibutylamino groups causes significant emission solvatochromism. Furthermore, red-shifted broad structureless bands are observed in polar solvents and this observation is characteristic of intramolecular charge transfer in the excited states. The electrochemical results demonstrate that, with the exception of compound $\mathbf{5 c}$, all systems can support stable radical cations and show their potential as hole-transporting materials.

\section{Experimental Section}

General. All reagents obtained from commercial sources were used as received. In air- and moisture-sensitive reactions, all glassware was flame-dried and cooled under argon. Anhydrous solvents were distilled over the appropriate drying agent. The DME/water mixtures used in Suzuki reactions were degassed by three freeze-pump-thaw cycles. Microwave-assisted reactions were carried out in a CEM Discover ${ }^{\mathrm{TM}}$ single mode microwave equipped with a controller for temperature, pressure, applied power, and with stirring of the treated suspension. NMR spectra were acquired at room temperature. NMR chemical shifts ( $\delta)$ are given in ppm relative to residual $\mathrm{CHCl}_{3}$ at $7.27 \mathrm{ppm}\left({ }^{1} \mathrm{H}\right), \mathrm{CDCl}_{3}$ at $77.0 \mathrm{ppm}\left({ }^{13} \mathrm{C}\right)$ and $\mathrm{CFCl}_{3}$ at $0.0 \mathrm{ppm}\left({ }^{19} \mathrm{~F}\right.$, external standard). Acidic impurities in $\mathrm{CDCl}_{3}$ were removed by treatment with anhydrous $\mathrm{K}_{2} \mathrm{CO}_{3}$. IR spectra were recorded on an FT-IR spectrophotometer equipped with an ATR accessory. MALDI-TOF mass spectra were registered in positive detection mode. Melting points are uncorrected. UV-vis and fluorescence spectra were recorded using standard $1 \mathrm{~cm}$ quartz cells. The $\Phi_{\text {F }}$ values were calculated using a well-known procedure with two different standards, quinine sulfate in $0.1 \mathrm{M} \mathrm{H}_{2} \mathrm{SO}_{4}$ and 9,10-diphenylanthracene in cyclohexane. ${ }^{[13]}$ Cyclic voltammetry measurements were performed using a glassy carbon working electrode, Pt counter electrode, and $\mathrm{Ag} / \mathrm{AgCl}$ reference electrode. The experiments were carried out under argon in $\mathrm{DCM}$, with $\mathrm{Bu}_{4} \mathrm{NPF}_{6}$ as supporting electrolyte $\left(0.1 \mathrm{~mol} \mathrm{~L}^{-1}\right)$. The scan rate was $100 \mathrm{mV} \mathrm{s}^{-1}$. Diethyl 4-(trifluoromethyl)benzylphosphonate and diethyl 4-tert-butylbenzylphosphonate were obtained by Arbuzov reaction of the appropriate bromide derivative with triethyl phosphite following a standard methodology (see compound 6).

Calculation methods. All calculations included in this work were optimized using Density Functional Theory (DFT) calculations with the 6$31 \mathrm{G}^{\left.*^{[14]}\right]}$ basis set, employing Becke's three parameterised Lee-YangParr exchange functional (B3LYP) ${ }^{[15]}$ and using the Gaussian 09 Program suite. ${ }^{[16]}$ Frequency calculations were performed to confirm the nature of the stationary points and to obtain zero-point energies (ZPEs). The frontier molecular orbitals were calculated with $6-31 G^{*}$ using Gaussview to visualize the topologies.

3,3'-Bis(4-formylphenyl)-2,2'-bithiophene (1). A mixture of 3,3'dibromo-2,2'-bithiophene $(2.00 \mathrm{~g}, 6.17 \mathrm{mmol})$, 4-formylphenylboronic acid (2.31 g, $15.4 \mathrm{mmol})$, and tetrakis(triphenylphosphine)palladium(0) (347 $\mathrm{mg}, 0.31 \mathrm{mmol})$ in degassed DME/aqueous $2 \mathrm{M}$ sodium carbonate $(3: 2$, $50 \mathrm{~mL}$ ) was heated at $100^{\circ} \mathrm{C}$ for $24 \mathrm{~h}$ under argon. The mixture was cooled and the DME was removed under reduced pressure. The product was extracted with DCM $(\times 3)$. The combined organic layers were dried $\left(\mathrm{MgSO}_{4}\right)$, concentrated, and filtered through a short chromatography column $\left(\mathrm{SiO}_{2}\right)$. The solvent was evaporated and the product was purified by washing with boiling EtOAc/EtOH (1:1). White solid (1.57 g, 68\%). Further purification was achieved by crystallization from $\mathrm{CHCl}_{3} / \mathrm{EtOH}$.
M.p. $190-192{ }^{\circ} \mathrm{C} ;{ }^{1} \mathrm{H}$ NMR $\left(500 \mathrm{MHz}, \mathrm{CDCl}_{3}\right): \delta=7.04(\mathrm{~d}, 4 \mathrm{H}, \mathrm{J}=8.0 \mathrm{~Hz}$, $\mathrm{ArH}), 7.10(\mathrm{~d}, 2 \mathrm{H}, \mathrm{J}=5.5 \mathrm{~Hz}, 2 \times \mathrm{CH}$ thiophene $), 7.45(\mathrm{~d}, 2 \mathrm{H}, \mathrm{J}=5.5 \mathrm{~Hz}$, $2 \times \mathrm{CH}$ thiophene), $7.57(\mathrm{~d}, 4 \mathrm{H}, \mathrm{J}=8.0 \mathrm{~Hz}, \mathrm{ArH}), 9.92(\mathrm{~s}, 2 \mathrm{H}, 2 \times \mathrm{CH}=\mathrm{O})$; ${ }^{13} \mathrm{C}$ NMR and DEPT (125 MHz, $\left.\mathrm{CDCl}_{3}\right): \delta=191.7(\mathrm{CH}=\mathrm{O}), 141.9(\mathrm{C})$, $139.8(\mathrm{C}), 134.5(\mathrm{C}), 131.1(\mathrm{C}), 129.5(\mathrm{CH}), 129.2(\mathrm{CH}), 128.7(\mathrm{CH})$, $127.0(\mathrm{CH})$; IR (ATR): $v=1688(\mathrm{C}=\mathrm{O}), 1601,1211,1161,829,804,739$ $\mathrm{cm}^{-1}$; MALDI-TOF (no matrix): $\mathrm{m} / \mathrm{z} 374.0[\mathrm{M}]^{+}$; elemental analysis calcd (\%) for $\mathrm{C}_{22} \mathrm{H}_{14} \mathrm{O}_{2} \mathrm{~S}_{2}$ : C 70.56, H 3.77, S 17.13; found: C 70.32, H 3.77. 5,5'-Dibromo-3,3'-bis(4-formylphenyl)-2,2'-bithiophene (2). To a stirred solution of 1 (1 g, $2.67 \mathrm{mmol})$ in anhydrous DMF (30 mL) was added NBS (1.43 g, $8.01 \mathrm{mmol})$ in small portions. The mixture was stirred in the dark at room temperature for $40 \mathrm{~h}$. Water $(200 \mathrm{~mL})$ was added and the precipitate was filtered off and washed with $\mathrm{MeOH}$. White solid $(1.36 \mathrm{~g}, 96 \%)$. Further purification was achieved by crystallization from $\mathrm{CHCl}_{3} / \mathrm{EtOH}$. M.p. $231-233^{\circ} \mathrm{C} ;{ }^{1} \mathrm{H} \mathrm{NMR}\left(500 \mathrm{MHz}, \mathrm{CDCl}_{3}\right): \delta=7.03$ $(\mathrm{d}, 4 \mathrm{H}, \mathrm{J}=8.0 \mathrm{~Hz}, \mathrm{ArH}), 7.05(\mathrm{~s}, 2 \mathrm{H}, 2 \times \mathrm{CH}$ thiophene), $7.61(\mathrm{~d}, 4 \mathrm{H}, \mathrm{J}=$ $8.0 \mathrm{~Hz}, \mathrm{ArH}), 9.94(\mathrm{~s}, 2 \mathrm{H}, 2 \times \mathrm{CH}=\mathrm{O}) ;{ }^{13} \mathrm{C}$ NMR and DEPT $(125 \mathrm{MHz}$, $\left.\mathrm{CDCl}_{3}\right): \delta=191.5(\mathrm{CH}=\mathrm{O}), 140.8(\mathrm{C}), 140.3(\mathrm{C}), 135.0(\mathrm{C}), 131.9(\mathrm{CH})$, $131.0(\mathrm{C}), 129.6(\mathrm{CH}), 128.7(\mathrm{CH}), 114.4(\mathrm{C}-\mathrm{Br})$; IR (ATR): $v=1682$ $(\mathrm{C}=\mathrm{O}), 1603,1395,1211,1161,825,808 \mathrm{~cm}^{-1}$; MALDI-TOF (dithranol): $\mathrm{m} / \mathrm{z} 532.0[\mathrm{M}+2]^{+\cdot}, 530.0\left[\mathrm{M}^{+*}\right.$; elemental analysis calcd (\%) for $\mathrm{C}_{22} \mathrm{H}_{12} \mathrm{Br}_{2} \mathrm{O}_{2} \mathrm{~S}_{2}$ : C 49.64, H 2.27, Br 30.02, S 12.05; found: C 49.41, H 2.76 .

Compound 3a. Solid $\mathrm{K}^{\mathrm{t}} \mathrm{BuO}$ (555 mg, $4.95 \mathrm{mmol}$ ) was added in small portions to a solution of diethyl 4-(trifluoromethyl)benzylphosphonate (488 $\mathrm{mg}, 1.65 \mathrm{mmol})$ in dry THF $(15 \mathrm{~mL})$ at $0{ }^{\circ} \mathrm{C}$ under argon. After 10 $\mathrm{min}$, the red mixture was cooled to $-78^{\circ} \mathrm{C}$ and a solution of dialdehyde 2 (400 mg, $0.75 \mathrm{mmol})$ in THF (10 mL) was added dropwise. The mixture was stirred at $-78^{\circ} \mathrm{C}$ for $1 \mathrm{~h}$ and quenched with saturated aqueous ammonium chloride at that temperature. The THF was evaporated, water was added, and the precipitate was filtered off and washed with $\mathrm{MeOH}$. Yellowish solid (550 mg, 90\%). Further purification was achieved by crystallization from THF/MeOH. M.p. decomposes $>228{ }^{\circ} \mathrm{C} ;{ }^{1} \mathrm{H}$ NMR $\left(500 \mathrm{MHz}, \mathrm{CDCl}_{3}\right): \delta=7.05\left(\mathrm{~A}\right.$ of $\left.\mathrm{AB}_{\mathrm{q}}, 4 \mathrm{H}, \mathrm{J}=8.0 \mathrm{~Hz}, \mathrm{ArH}\right), 7.08(\mathrm{~s}, 2 \mathrm{H}$ $2 \times \mathrm{CH}$ thiophene), $7.08\left(\mathrm{~A}\right.$ of $\left.\mathrm{AB}_{\mathrm{q}}, 2 \mathrm{H}, \mathrm{J}=16.0 \mathrm{~Hz}, 2 \times \mathrm{CH}=\right), 7.14(\mathrm{~B}$ of $\left.A_{\mathrm{q}}, 2 \mathrm{H}, \mathrm{J}=16.0 \mathrm{~Hz}, 2 \times \mathrm{CH}=\right), 7.34\left(\mathrm{~B}\right.$ of $\left.\mathrm{AB}_{\mathrm{q}}, 4 \mathrm{H}, \mathrm{J}=8.0 \mathrm{~Hz}, \mathrm{ArH}\right)$, $7.59\left(A\right.$ of $\left.A B_{q}, 4 \mathrm{H}, J=8.5 \mathrm{~Hz}, A r H\right), 7.62\left(B\right.$ of $A B_{q}, 4 H, J=8.5 \mathrm{~Hz}$, $\mathrm{ArH}) ;{ }^{13} \mathrm{C}$ NMR and DEPT $\left(125 \mathrm{MHz}, \mathrm{CDCl}_{3}\right): \delta=141.6(\mathrm{C}), 140.6(\mathrm{C})$, $135.6(\mathrm{C}), 134.4(\mathrm{C}), 131.9(\mathrm{CH}), 130.5(\mathrm{CH}), 130.2(\mathrm{C}), 129.4$ (q, J = $32.5 \mathrm{~Hz}, \mathrm{C}), 128.6(\mathrm{CH}), 127.3(\mathrm{CH}), 126.7(\mathrm{CH}), 126.6(\mathrm{CH}), 125.7(\mathrm{q}, \mathrm{J}$ $=3.8 \mathrm{~Hz}, \mathrm{CH}), 124.2(\mathrm{q}, \mathrm{J}=270.9 \mathrm{~Hz}, \mathrm{C}), 113.6(\mathrm{C})$; IR (ATR): $v=1319$, 1123, 1109, 1067, $831 \mathrm{~cm}^{-1}$; MALDI-TOF (dithranol): $\mathrm{m} / \mathrm{z} 815.6[\mathrm{M}+2]^{+}$, $813.6[\mathrm{M}]^{+*}, 735.9[\mathrm{M}-\mathrm{Br}]^{+*}$; elemental analysis calcd (\%) for $\mathrm{C}_{38} \mathrm{H}_{22} \mathrm{Br}_{2} \mathrm{~F}_{6} \mathrm{~S}_{2}$ : C 55.90, H 2.72, Br 19.57, F 13.96, S, 7.85; found: C 55.64, H 2.99 .

Compound 3b. This compound was prepared from $\mathrm{K}^{t} \mathrm{BuO}$ (527 mg, 4.70 mmol), dialdehyde 2 (500 mg, $0.94 \mathrm{mmol})$, and diethyl 4-tertbutylbenzylphosphonate ( $802 \mathrm{mg}, 2.82 \mathrm{mmol}$ ) using the same procedure as described for $\mathbf{3 a}$. The product was purified by washing with boiling EtOH. White solid (501 mg, 67\%). M.p. decomposes $>248{ }^{\circ} \mathrm{C} ;{ }^{1} \mathrm{H}$ NMR $\left(500 \mathrm{MHz}, \mathrm{CDCl}_{3}\right): \delta=1.34\left(\mathrm{~s}, 18 \mathrm{H}, 6 \times \mathrm{CH}_{3}\right), 6.99\left(\mathrm{~A}\right.$ of $\mathrm{AB}_{\mathrm{q}}, 4 \mathrm{H}, \mathrm{J}=8.0$ $\mathrm{Hz}, \mathrm{ArH}), 7.00\left(\mathrm{~A}\right.$ of $\left.\mathrm{AB}_{\mathrm{q}}, 2 \mathrm{H}, \mathrm{J}=16.0 \mathrm{~Hz}, 2 \times \mathrm{CH}=\right), 7.05(\mathrm{~s}, 2 \mathrm{H}, \mathrm{CH}$ thiophene), 7.06 ( $B$ of $\left.A B_{q}, 2 H, J=16.0 \mathrm{~Hz}, 2 \times C H=\right), 7.29\left(B\right.$ of $A B_{q}, 4 H$, $\mathrm{J}=8.0 \mathrm{~Hz}, \mathrm{ArH}), 7.39\left(\mathrm{~A}\right.$ of $\left.\mathrm{AB}_{\mathrm{c}}, 4 \mathrm{H}, \mathrm{J}=8.5 \mathrm{~Hz}, \mathrm{ArH}\right), 7.45\left(\mathrm{~B}\right.$ of $\mathrm{AB}_{\mathrm{c}}, 4 \mathrm{H}$, $\mathrm{J}=8.5 \mathrm{~Hz}, \mathrm{ArH}) ;{ }^{13} \mathrm{C}$ and DEPT $\left(125 \mathrm{MHz}, \mathrm{CDCl}_{3}\right): \delta=150.9(\mathrm{C}), 141.8$ $(\mathrm{C}), 136.6(\mathrm{C}), 134.4(\mathrm{C}), 133.5(\mathrm{C}), 131.9(\mathrm{CH}), 130.0(\mathrm{C}), 128.7(\mathrm{CH})$, $128.5(\mathrm{CH}), 127.3(\mathrm{CH}), 126.3(\mathrm{CH}), 125.6(\mathrm{CH}), 113.3(\mathrm{C}), 34.6(\mathrm{C})$ $31.3\left(\mathrm{CH}_{3}\right) ; \mathrm{IR}$ (ATR): $v=1487,1109,964,826 \mathrm{~cm}^{-1}$; MALDI-TOF (dithranol): $\mathrm{m} / \mathrm{z} 791.8[\mathrm{M}+2]^{+}, 779.8[\mathrm{M}]^{+\cdot}, 712[\mathrm{M}-\mathrm{Br}]^{+*}$; elemental analysis calcd (\%) for $\mathrm{C}_{44} \mathrm{H}_{40} \mathrm{Br}_{2} \mathrm{~S}_{2}$ : C 66.66, H 5.09, $\mathrm{Br} 20.16$, S 8.09; found: C 66.55, H, 5.20.

Compound $\mathbf{4 a}$. This compound was prepared from compound $\mathbf{3 a}$ (290 $\mathrm{mg}, 0.36 \mathrm{mmol}$ ), 4-formylphenylboronic acid (133 $\mathrm{mg}, 0.89 \mathrm{mmol}$ ), and tetrakis(triphenylphosphine)palladium(0) (28 $\mathrm{mg}, 0.018 \mathrm{mmol}$ ) using the same procedure as described for 1 . The product was purified by column 
chromatography $\left(\mathrm{SiO}_{2}, \mathrm{CHCl}_{3}\right)$ followed by crystallization from $\mathrm{CHCl}_{3} / \mathrm{MeOH}$. Yellow solid $255 \mathrm{mg}(83 \%) .{ }^{1} \mathrm{H}$ NMR $\left(500 \mathrm{MHz}, \mathrm{CDCl}_{3}\right): \delta$ $=7.08\left(\mathrm{~A}\right.$ of $\left.\mathrm{AB}_{\mathrm{q}}, 2 \mathrm{H}, \mathrm{J}=16.0 \mathrm{~Hz}, 2 \times \mathrm{CH}=\right), 7.12\left(\mathrm{~A}\right.$ of $\mathrm{AB}_{\mathrm{q}}, 4 \mathrm{H}, \mathrm{J}=8.0$ $\mathrm{Hz}, \mathrm{ArH}), 7.15\left(\mathrm{~B}\right.$ of $\left.\mathrm{AB}_{\mathrm{q}}, 2 \mathrm{H}, \mathrm{J}=16.0 \mathrm{~Hz}, 2 \times \mathrm{CH}=\right), 7.34(\mathrm{~B}$ of $\mathrm{AB}, 4 \mathrm{H}$, $\mathrm{J}=8.0 \mathrm{~Hz}, \mathrm{ArH}), 7.49(\mathrm{~s}, 2 \mathrm{H}, 2 \times \mathrm{CH}$ thiophene), $7.59(\mathrm{~A}$ of $\mathrm{AB}, 4 \mathrm{H}, \mathrm{J}=$ $8.5 \mathrm{~Hz}, \mathrm{ArH}), 7.62\left(\mathrm{~B}\right.$ of $\left.\mathrm{AB}_{\mathrm{q}}, 4 \mathrm{H}, \mathrm{J}=8.5 \mathrm{~Hz}, \mathrm{ArH}\right), 7.79\left(\mathrm{~A}_{\mathrm{A}} \mathrm{AB}, 4 \mathrm{H}, \mathrm{J}\right.$ $=8.5 \mathrm{~Hz}, \mathrm{ArH}), 7.93\left(\mathrm{~B}\right.$ of $\left.\mathrm{AB}_{\mathrm{q}}, 4 \mathrm{H}, \mathrm{J}=8.5 \mathrm{~Hz}, \mathrm{ArH}\right), 10.03(\mathrm{~s}, 2 \mathrm{H}, 2 \times$ $\mathrm{CH}=\mathrm{O}) ;{ }^{13} \mathrm{C}$ NMR and DEPT $\left(125 \mathrm{MHz}, \mathrm{CDCl}_{3}\right): \delta=191.3(\mathrm{CH}=\mathrm{O}), 143.0$ (C), 142.2 (C), 140.6 (C), 139.2 (C), 135.5 (C), 135.3 (C), 131.0 (C), $130.6(\mathrm{CH}), 130.5(\mathrm{CH}), 129.4(\mathrm{q}, \mathrm{J}=31.9 \mathrm{~Hz}, \mathrm{C}), 128.7(\mathrm{CH}), 127.2$ $(\mathrm{CH}), 127.2(\mathrm{CH}), 126.6(\mathrm{CH}), 126.5(\mathrm{CH}), 125.8(\mathrm{CH}), 125.7(\mathrm{q}, \mathrm{J}=3.9$ $\mathrm{Hz}, \mathrm{CH}), 124.2$ (q, J = 270.1 Hz, $\mathrm{CF}_{3}$ ); IR (ATR): $v=1693$ (C=O), 1599, 1321, 1169, 1065, $822 \mathrm{~cm}^{-1}$; MALDI-TOF (dithranol): $\mathrm{m} / \mathrm{z} 865.7[\mathrm{M}]^{+}$; elemental analysis calcd (\%) for $\mathrm{C}_{52} \mathrm{H}_{32} \mathrm{~F}_{6} \mathrm{O}_{2} \mathrm{~S}_{2}: \mathrm{C} 72.04, \mathrm{H} 3.72, \mathrm{~F} 13.15$, S 7.40; found: C $72.25, \mathrm{H}, 3.70$

Compound $\mathbf{4 b}$. This compound was prepared from compound $\mathbf{3 b}$ (405 $\mathrm{mg}, 0.51 \mathrm{mmol}), 4$-formylphenylboronic acid (192 mg, $1.28 \mathrm{mmol})$, and tetrakis(triphenylphosphine)palladium(0) $(40 \mathrm{mg}, 0.025 \mathrm{mmol})$ using the same procedure as described for $\mathbf{1}$. The crude product was filtered through Celite and purified by crystallization from EtOAc/hexanes. Yellow solid $327 \mathrm{mg}(76 \%) .{ }^{1} \mathrm{H}$ NMR $\left(500 \mathrm{MHz}, \mathrm{CDCl}_{3}\right): \delta=1.34(\mathrm{~s}, 18 \mathrm{H}, 6 \times$ $\left.\mathrm{CH}_{3}\right), 7.01\left(\mathrm{~A}\right.$ of $\left.\mathrm{AB}_{\mathrm{q}}, 2 \mathrm{H}, \mathrm{J}=16.5 \mathrm{~Hz}, 2 \times \mathrm{CH}=\right), 7.06(\mathrm{~B}$ of $\mathrm{AB}, 2 \mathrm{H}, J=$ $16.0 \mathrm{~Hz}, 2 \times \mathrm{CH}=), 7.07\left(\mathrm{~A}\right.$ of $\left.A B_{\mathrm{q}}, 4 \mathrm{H}, J=8.5 \mathrm{~Hz}, \mathrm{ArH}\right), 7.29\left(\mathrm{~B}\right.$ of $A B_{\mathrm{q}}$, $4 \mathrm{H}, J=8.5 \mathrm{~Hz}, \mathrm{ArH}), 7.39\left(\mathrm{~A}\right.$ of $\left.\mathrm{AB}_{\mathrm{q}}, 4 \mathrm{H}, \mathrm{J}=8.5 \mathrm{~Hz}, \mathrm{ArH}\right), 7.45$ (B of $\mathrm{AB}_{\mathrm{r}}$ $4 \mathrm{H}, J=8.5 \mathrm{~Hz}, \mathrm{ArH}), 7.47\left(\mathrm{~s}, 2 \mathrm{H}, 2 \times \mathrm{CH}\right.$ thiophene), $7.78\left(\mathrm{~A}\right.$ of $\mathrm{AB}_{\mathrm{q}}, 4 \mathrm{H}$, $\mathrm{J}=8.5 \mathrm{~Hz}, \mathrm{ArH}), 7.91\left(\mathrm{~B}\right.$ of $\left.\mathrm{AB}_{\mathrm{q}}, 4 \mathrm{H}, \mathrm{J}=8.5 \mathrm{~Hz}, \mathrm{ArH}\right), 10.02(\mathrm{~s}, 2 \mathrm{H}, 2 \times$ $\mathrm{CH}=\mathrm{O}) ;{ }^{13} \mathrm{C}$ NMR and DEPT $\left(125 \mathrm{MHz}, \mathrm{CDCl}_{3}\right): \delta=191.4(\mathrm{CH}=\mathrm{O}), 151.0$ (C), 142.8 (C), 142.4 (C), 139.3 (C), 136.4 (C), 135.4 (C), 134.4 (C), $134.3(\mathrm{C}), 130.9(\mathrm{C}), 130.5(\mathrm{CH}), 128.6(\mathrm{CH}), 128.5(\mathrm{CH}), 127.4(\mathrm{CH})$, $127.3(\mathrm{CH}), 126.2(\mathrm{CH}), 126.2(\mathrm{CH}), 125.8(\mathrm{CH}), 125.7(\mathrm{CH}), 34.6(\mathrm{C})$, $31.3\left(\mathrm{CH}_{3}\right)$; IR (ATR): $v=1697(\mathrm{C}=\mathrm{O}), 1599,1217,1169,964,820 \mathrm{~cm}^{-1}$; MALDI-TOF (dithranol): $\mathrm{m} / \mathrm{z} 842.1[\mathrm{M}]^{+}$; elemental analysis calcd (\%) for $\mathrm{C}_{58} \mathrm{H}_{50} \mathrm{O}_{2} \mathrm{~S}_{2}$ : C 82.62, H 5.98, S 7.60; found: C 82.43, H, 5.91.

Compound 5a. Solid $\mathrm{K}^{\mathrm{t}} \mathrm{BuO}(95 \mathrm{mg}, 0.85 \mathrm{mmol}$ ) was added in small portions to a solution of dialdehyde $4 a(150 \mathrm{mg}, 0.17 \mathrm{mmol})$ and diethyl 4-tert-butylbenzylphosphonate (145 mg, $0.51 \mathrm{mmol})$ in dry THF $(5 \mathrm{~mL})$ under argon. The mixture was stirred at room temperature for $3 \mathrm{~h}$ and quenched with water. The THF was evaporated and the precipitate was filtered off. The product was purified by column chromatography $\left(\mathrm{SiO}_{2}\right.$, hexanes/EtOAc 6:4) followed by crystallization from $\mathrm{CHCl}_{3} / \mathrm{MeOH}$. Orange solid $62 \mathrm{mg}(32 \%) .{ }^{1} \mathrm{H}$ NMR $\left(500 \mathrm{MHz}, \mathrm{CDCl}_{3}\right): \delta=1.34(\mathrm{~s}, 18 \mathrm{H}$, $\left.6 \times \mathrm{CH}_{3}\right), 7.06\left(\mathrm{~A}\right.$ of $\left.\mathrm{AB}_{\mathrm{q}}, 2 \mathrm{H}, \mathrm{J}=16.5 \mathrm{~Hz}, 2 \times \mathrm{CH}=\right), 7.08(\mathrm{~A}$ of $\mathrm{AB}, 2 \mathrm{H}, \mathrm{J}$ $=16.5 \mathrm{~Hz}, 2 \times \mathrm{CH}=), 7.14\left(\mathrm{~A}_{\text {of }} \mathrm{AB}_{\mathrm{q}}, 4 \mathrm{H}, J=8.5 \mathrm{~Hz}, \mathrm{ArH}\right), 7.14(2 \times \mathrm{B}$ of $\left.\mathrm{AB}_{\mathrm{q}}, 4 \mathrm{H}, J=16.5 \mathrm{~Hz}, 4 \times \mathrm{CH}=\right), 7.32\left(\mathrm{~B}\right.$ of $\left.\mathrm{AB}_{\mathrm{q}}, 4 \mathrm{H}, J=8.5 \mathrm{~Hz}, \mathrm{ArH}\right)$ 7.35 (s, $2 \mathrm{H}, 2 \times \mathrm{CH}$ thiophene), 7.40 ( $\mathrm{A}$ of $\mathrm{AB}_{\mathrm{q}}, 4 \mathrm{H}, \mathrm{J}=8.5 \mathrm{~Hz}, \mathrm{ArH}$ ), 7.47 ( $B$ of $\left.A B_{q}, 4 \mathrm{H}, J=8.5 \mathrm{~Hz}, A r H\right), 7.53\left(A\right.$ of $\left.A B_{q}, 4 \mathrm{H}, J=8.5 \mathrm{~Hz}, A r H\right)$, $7.58\left(A\right.$ of $\left.A B_{q}, 4 \mathrm{H}, J=9.0 \mathrm{~Hz}, \mathrm{ArH}\right), 7.60\left(\mathrm{~B}\right.$ of $\left.A B_{q}, 4 \mathrm{H}, J=9.0 \mathrm{~Hz}, \mathrm{ArH}\right)$ $7.61\left(B\right.$ of $\left.A B_{q}, 4 \mathrm{H}, J=8.5 \mathrm{~Hz}, \mathrm{ArH}\right) ;{ }^{13} \mathrm{C}$ NMR and DEPT (125 MHz, $\left.\mathrm{CDCl}_{3}\right): \delta=151.0$ (C), 144.3 (C), 141.5 (C), 140.8 (C), 137.3 (C), 135.9 (C), 135.1 (C), 134.4 (C), 132.6 (C), $130.9(\mathrm{CH}), 129.4$ (C), 129.3 (q, J = $32.6 \mathrm{~Hz}, \mathrm{C}), 128.9(\mathrm{CH}), 128.7(\mathrm{CH}), 127.1(\mathrm{CH}), 127.0(\mathrm{CH}), 126.9(\mathrm{CH})$ $126.6(\mathrm{CH}), 126.5(\mathrm{CH}), 126.3(\mathrm{CH}), 125.8(\mathrm{CH}), 125.7(\mathrm{CH}), 125.7(\mathrm{q}, \mathrm{J}$ $=3.8 \mathrm{~Hz}, \mathrm{CH}), 125.0(\mathrm{CH}), 124.2\left(\mathrm{q}, \mathrm{J}=270.1 \mathrm{~Hz}, \mathrm{CF}_{3}\right), 34.7(\mathrm{C}), 31.3$ $\left(\mathrm{CH}_{3}\right)$; MALDI-TOF (dithranol) $\mathrm{m} / \mathrm{z}$ : $1126.1[\mathrm{M}]^{+}$; elemental analysis calcd (\%) for $\mathrm{C}_{74} \mathrm{H}_{60} \mathrm{~F}_{6} \mathrm{~S}_{2}$ : C 78,84, H 5.36, F 10.11, S 5,69; found: C 78.90, H 5.45 .

Compound 5b. This compound was prepared from $\mathrm{K}^{t} \mathrm{BuO}(101 \mathrm{mg}, 0.90$ $\mathrm{mmol})$, dialdehyde 4b $(150 \mathrm{mg}, 0.18 \mathrm{mmol})$, and diethyl 4(trifluoromethyl)benzylphosphonate (158 $\mathrm{mg}, 0.53 \mathrm{mmol}$ ) using the same procedure as described for $\mathbf{5 a}$. The product was purified by column chromatography $\left(\mathrm{SiO}_{2}\right.$, hexanes/EtOAc $\left.7: 3\right)$ followed by crystallization from $\mathrm{CHCl}_{3} / \mathrm{MeOH}$. Yellow solid $91 \mathrm{mg}(45 \%)$. M.p. decomposes $>290$ ${ }^{\circ} \mathrm{C} ;{ }^{1} \mathrm{H}$ NMR $\left(500 \mathrm{MHz}, \mathrm{CDCl}_{3}\right): \delta=1.34\left(\mathrm{~s}, 18 \mathrm{H}, 6 \times \mathrm{CH}_{3}\right), 7.01\left(\mathrm{~A}^{\circ} \mathrm{AB}_{\mathrm{q}}\right.$ $2 \mathrm{H}, J=16.5 \mathrm{~Hz}, 2 \times \mathrm{CH}=), 7.06\left(\mathrm{~B}\right.$ of $\left.\mathrm{AB}_{\mathrm{q}}, 2 \mathrm{H}, \mathrm{J}=16.5 \mathrm{~Hz}, 2 \times \mathrm{CH}=\right)$, $7.10\left(A\right.$ of $\left.A B_{q}, 4 H, J=8.0 \mathrm{~Hz}, A r H\right), 7.14\left(A\right.$ of $A B_{q}, 2 H, J=16.5 \mathrm{~Hz}, 2 \times$
$\mathrm{CH}=), 7.20\left(\mathrm{~B}\right.$ of $\left.\mathrm{AB}_{\mathrm{q}}, 2 \mathrm{H}, \mathrm{J}=16.5 \mathrm{~Hz}, 2 \times \mathrm{CH}=\right), 7.29\left(\mathrm{~B}\right.$ of $\mathrm{AB}_{\mathrm{q}}, 4 \mathrm{H}, \mathrm{J}=$ $8.0 \mathrm{~Hz}, \mathrm{ArH}), 7.37\left(\mathrm{~s}, 2 \mathrm{H}, 2 \times \mathrm{CH}\right.$ thiophene), $7.39\left(\mathrm{~A}\right.$ of $\mathrm{AB}_{\mathrm{q}}, 4 \mathrm{H}, \mathrm{J}=8.5$ $\mathrm{Hz}, \mathrm{ArH}$ ), 7.45 (B of $\left.A B_{q}, 4 \mathrm{H}, J=8.5 \mathrm{~Hz}, \mathrm{ArH}\right), 7.55$ ( $\mathrm{A}$ of $A B_{q}, 4 \mathrm{H}, \mathrm{J}=$ $8.0 \mathrm{~Hz}, \mathrm{ArH}), 7.61(\mathrm{~s}, 8 \mathrm{H}, \mathrm{ArH}), 7.63(\mathrm{~B}$ of $\mathrm{AB}, 4 \mathrm{H}, \mathrm{J}=8.0 \mathrm{~Hz}, \mathrm{ArH}) ;{ }^{13} \mathrm{C}$ NMR and DEPT (125 MHz, CDCl $): \delta=150.8$ (C), 143.8 (C), 141.9 (C), 140.7 (C), 136.1 (C), 136.1 (C), 134.9 (C), 134.5 (C), 133.6 (C), 130.5 $(\mathrm{CH}), 129.5(\mathrm{C}), 129.3(\mathrm{q}, \mathrm{J}=32.6 \mathrm{~Hz}, \mathrm{C}), 128.6(\mathrm{CH}), 128.3(\mathrm{CH}), 127.6$ $(\mathrm{CH}), 127.4(\mathrm{CH}), 127.3(\mathrm{CH}), 126.6(\mathrm{CH}), 126.2(\mathrm{CH}), 126.2(\mathrm{CH}), 125.8$ $(\mathrm{CH}), 125.7(\mathrm{q}, \mathrm{J}=3.8 \mathrm{~Hz}, \mathrm{CH}), 125.6(\mathrm{CH}), 125.4(\mathrm{CH}), 124.2$ (q, $\mathrm{J}=$ $\left.270.1 \mathrm{~Hz}, \mathrm{CF}_{3}\right), 34.6(\mathrm{C}), 31.3\left(\mathrm{CH}_{3}\right) ;{ }^{19} \mathrm{~F}$ NMR $\left(282 \mathrm{MHz}, \mathrm{CDCl}_{3}\right): \delta=$ -62.9 ; IR (ATR): $v=1612,1321,1161,1121,1065,966,831 \mathrm{~cm}^{-1}$; MALDI-TOF (dithranol): $\mathrm{m} / \mathrm{z} 1126.5$ [M] $^{+}$; elemental analysis calcd (\%) for $\mathrm{C}_{74} \mathrm{H}_{60} \mathrm{~F}_{6} \mathrm{~S}_{2}$ : C 78,84, H 5.36, F 10.11, S 5,69; found: C 78.71, H, 5.40. Phosponate 6. A mixture of commercially available 4bromomethylphenylboronic acid pinacol ester $(900 \mathrm{mg}, 3.03 \mathrm{mmol})$ and triethyl phosphite $\left(3 \mathrm{~mL}\right.$, excess) was heated at $140{ }^{\circ} \mathrm{C}$ for $1 \mathrm{~h}$. The excess reagent was distilled off under reduced pressure (Kugelrohr) Phosphonate $\mathbf{6}$ was obtained as colorless viscous oil that solidified upon standing $(1.07 \mathrm{~g}, 100 \%)$ and this was used in the next step. ${ }^{1} \mathrm{H}$ NMR (500 $\left.\mathrm{MHz}, \mathrm{CDCl}_{3}\right): \delta=1.24\left(\mathrm{t}, 6 \mathrm{H}, J=7.0 \mathrm{~Hz}, 2 \times \mathrm{CH}_{3}\right), 1.34\left(\mathrm{~s}, 12 \mathrm{H}, 4 \times \mathrm{CH}_{3}\right)$, $3.17\left(\mathrm{~d}, 2 \mathrm{H}, \mathrm{J}=22.0 \mathrm{~Hz}, \mathrm{CH}_{2} \mathrm{P}\right), 4.00\left(\mathrm{~m}, 4 \mathrm{H}, 2 \times \mathrm{OCH}_{2}\right), 7.30(\mathrm{dd}, 2 \mathrm{H}, \mathrm{J}$ $=8.0 \mathrm{~Hz}, \mathrm{~J}=2.5 \mathrm{~Hz}, \mathrm{ArH}), 7.75(\mathrm{~d}, 2 \mathrm{H}, \mathrm{J}=8.0 \mathrm{~Hz}, \mathrm{ArH}) ;{ }^{13} \mathrm{C}$ NMR and DEPT (125 MHz, CDCl $): \delta=134.9(\mathrm{~d}, \mathrm{~J}=2.9 \mathrm{~Hz}, \mathrm{CH}), 134.9(\mathrm{~d}, \mathrm{~J}=9.6$ $\mathrm{Hz}, \mathrm{C}), 129.1(\mathrm{~d}, J=6.6 \mathrm{~Hz}, \mathrm{CH}), 83.8(\mathrm{C}), 62.1\left(\mathrm{~d}, J=6.6 \mathrm{~Hz}, \mathrm{OCH}_{2}\right)$ $34.1\left(\mathrm{~d}, \mathrm{~J}=136.4 \mathrm{~Hz}, \mathrm{CH}_{2} \mathrm{P}\right), 24.8\left(\mathrm{CH}_{3}\right), 16.3\left(\mathrm{~d}, \mathrm{~J}=5.7 \mathrm{~Hz}, \mathrm{CH}_{2} \mathrm{CH}_{3}\right)$ IR (ATR): $v=2978,1717,1610,1393,1358,1242,1144,1088,1051$, 1020, 962, 858, $658 \mathrm{~cm}^{-1}$; MALDI-TOF (dithranol): $\mathrm{m} / \mathrm{z} 355.1\left[\mathrm{M}+\mathrm{H}^{+}\right.$, $377.0\left[\mathrm{M}+\mathrm{Na}^{+}\right.$; elemental analysis calcd (\%) for $\mathrm{C}_{17} \mathrm{H}_{28} \mathrm{BO}{ }_{5} \mathrm{P}$ : C 57.65, H 7.97, B 3.05, P 8.75; found: C 57.50, H 8.01 .

Compound 7. This compound was prepared from $K^{t} \mathrm{BuO}(927 \mathrm{mg}, 8.26$ $\mathrm{mmol}), 4$-( $\mathrm{N}, \mathrm{N}$-dimethylamino)benzaldehyde $(937 \mathrm{mg}, 2.5 \mathrm{mmol})$, and phosphonate $6(974 \mathrm{mg}, 2.75 \mathrm{mmol})$ using the same procedure as described for $\mathbf{5 a}$. The mixture was stirred at room temperature for $3 \mathrm{~h}$ and quenched with saturated aqueous ammonium chloride. The THF was evaporated, water was added, and the mixture was extracted with $\mathrm{CH}_{2} \mathrm{Cl}_{2}(\times 3)$. The combined organic layers were dried $\left(\mathrm{MgSO}_{4}\right)$ and the solvent was evaporated. The crude product was purified by crystallization from $\mathrm{MeOH}$. Pale yellow crystals $(1.0 \mathrm{~g}, 93 \%)$. M.p. 92.0-93.5 ${ }^{\circ} \mathrm{C} ;{ }^{1} \mathrm{H}$ NMR (500 MHz, $\left.\mathrm{CDCl}_{3}\right): \delta=0.97\left(\mathrm{t}, 6 \mathrm{H}, \mathrm{J}=7.5 \mathrm{~Hz}, 2 \times \mathrm{CH}_{3}\right), 1.35(\mathrm{~s}$ $\left.12 \mathrm{H}, 4 \times \mathrm{CH}_{3}\right), 1.39\left(\mathrm{~m}, 4 \mathrm{H}, 2 \times \mathrm{CH}_{2} \mathrm{CH}_{2} \mathrm{CH}_{2} \mathrm{CH}_{3}\right), 1.59(\mathrm{~m}, 4 \mathrm{H}, 2 \times$ $\mathrm{CH}_{2} \mathrm{CH}_{2} \mathrm{CH}_{2} \mathrm{CH}_{3}$ ), 3.30 (t, $4 \mathrm{H}, J=7.5 \mathrm{~Hz}, 2 \times \mathrm{NCH}_{2}$ ), $6.63\left(\mathrm{~A}\right.$ of $\mathrm{AB}_{\mathrm{q}}, 2 \mathrm{H}$, $J=8.5 \mathrm{~Hz}, \mathrm{ArH}), 6.89\left(A\right.$ of $\left.A B_{\mathrm{q}}, 1 \mathrm{H}, J=16.5 \mathrm{~Hz}, \mathrm{CH}=\right), 7.10\left(B\right.$ of $A B_{\mathrm{q}}$, $1 \mathrm{H}, J=16.5 \mathrm{~Hz}, \mathrm{CH}=), 7.36\left(\mathrm{~B}\right.$ of $\left.\mathrm{AB}_{\mathrm{q}}, 2 \mathrm{H}, \mathrm{J}=8.5 \mathrm{~Hz}, \mathrm{ArH}\right), 7.47(\mathrm{~A}$ of $\left.\mathrm{AB}_{\mathrm{q}}, 2 \mathrm{H}, \mathrm{J}=8.0 \mathrm{~Hz}, \mathrm{ArH}\right), 7.77\left(\mathrm{~B}\right.$ of $\left.\mathrm{AB}_{\mathrm{q}}, 2 \mathrm{H}, \mathrm{J}=8.0 \mathrm{~Hz}, \mathrm{ArH}\right),{ }^{13} \mathrm{C}$ NMR $\left(125 \mathrm{MHz}, \mathrm{CDCl}_{3}\right): \delta=148.0,141.2,135.1,129.9,127.9,125.2,124.3$, 123.5, 111.6, 83.6 (C), $50.8\left(\mathrm{NCH}_{2}\right), 29.5\left(\mathrm{CH}_{2}\right), 24.9\left(\mathrm{CH}_{3}\right), 20.3\left(\mathrm{CH}_{2}\right)$, $14.0\left(\mathrm{CH}_{3}\right)$; IR (ATR): $v=1593,1512,1396,1356,1321,1182,1140$, 1088, 957, 858, 822, $654 \mathrm{~cm}^{-1}$; MALDI-TOF (dithranol): $\mathrm{m} / \mathrm{z} 323.2,433.3$ $[\mathrm{M}]^{+}$; elemental analysis calcd (\%) for $\mathrm{C}_{28} \mathrm{H}_{40} \mathrm{BNO}_{2}$ : C 77.59, $\mathrm{H}$ 9.30, B 2.49, N 3.23; found: C 77.71, H 9.27, N, 3.22.

Compound 5c. A microwave reactor vessel was loaded with a mixture of 3a (324 mg, $0.75 \mathrm{mmol}$ ), compound 7 (324 g, $0.75 \mathrm{mmol})$, and tetrakis(triphenylphosphine)palladium(0) $\quad(19 \mathrm{mg}, 0.017 \mathrm{mmol})$ in degassed DME/aqueous $2 \mathrm{M}$ sodium carbonate $(3: 2 \mathrm{v} / \mathrm{v}, 1 \mathrm{~mL})$ under argon. The vessel was sealed and the reaction mixture was stirred and irradiated at $150{ }^{\circ} \mathrm{C}$ (external surface sensor) for $30 \mathrm{~min}$ (power $120 \mathrm{~W}$, pressure $10 \mathrm{bar}$ ). The mixture was cooled, water was added, and the product was extracted with DCM $(\times 3)$. The combined organic layers were dried $\left(\mathrm{MgSO}_{4}\right)$, concentrated, and filtered through a short column of neutral alumina. The solvent was evaporated and the product was purified by washing with boiling $\mathrm{MeOH}$. Orange solid (330 mg, 77\%). Further purification was achieved by crystallization from THF/MeOH (9:1 v/v). M.p. $163.1-165.7^{\circ} \mathrm{C} ;{ }^{1} \mathrm{H} \mathrm{NMR}\left(500 \mathrm{MHz}, \mathrm{CDCl}_{3}\right): \delta=0.98(\mathrm{t}, 12 \mathrm{H}, \mathrm{J}$ $\left.=7.5 \mathrm{~Hz}, 4 \times \mathrm{CH}_{3}\right), 1.40\left(\mathrm{~m}, 8 \mathrm{H}, 4 \times \mathrm{CH}_{2}\right), 1.60\left(\mathrm{~m}, 8 \mathrm{H}, 4 \times \mathrm{CH}_{2}\right), 3.31(\mathrm{t}$, $8 \mathrm{H}, \mathrm{J}=7.5 \mathrm{~Hz}, 4 \times \mathrm{CH}_{2}$ ), $6.64\left(\mathrm{~A}_{\text {of }} \mathrm{AB}_{\mathrm{q}} 4 \mathrm{H}, \mathrm{J}=9.0 \mathrm{~Hz}, \mathrm{ArH}\right), 6.89$ (A of 
$\left.\mathrm{AB}_{\mathrm{q}} 2 \mathrm{H}, \mathrm{J}=16.0 \mathrm{~Hz}, 2 \times \mathrm{CH}=\right), 7.07\left(\mathrm{~A}\right.$ of $\left.\mathrm{AB}_{\mathrm{q}}, 2 \mathrm{H}, \mathrm{J}=16.5 \mathrm{~Hz}, 2 \times \mathrm{CH}=\right)$ $7.08\left(B\right.$ of $\left.A B_{q}, 2 \mathrm{H}, J=16.0 \mathrm{~Hz}, 2 \times C H=\right), 7.15\left(B\right.$ of $A B_{q}, 2 H, J=16.5$ $\mathrm{Hz}, 2 \times \mathrm{CH}=), 7.15\left(\mathrm{~A}\right.$ of $\left.\mathrm{AB}_{\mathrm{q}}, 4 \mathrm{H}, \mathrm{J}=8.5 \mathrm{~Hz}, \mathrm{ArH}\right), 7.32\left(B\right.$ of $A B_{\mathrm{q}}, 4 \mathrm{H}, \mathrm{J}$ $=8.5 \mathrm{~Hz}, \mathrm{ArH}), 7.34(\mathrm{~s}, 2 \mathrm{H}, 2 \times \mathrm{CH}$ thiophene), $7.40(\mathrm{~B}$ of $\mathrm{AB}, 4 \mathrm{H}, \mathrm{J}=$ $9.0 \mathrm{~Hz}, \mathrm{ArH}), 7.49\left(\mathrm{~A}\right.$ of $\left.A B_{\mathrm{q}}, 4 \mathrm{H}, \mathrm{J}=8.0 \mathrm{~Hz}, \mathrm{ArH}\right), 7.59\left(\mathrm{~B}\right.$ of $A B_{\mathrm{q}}, 4 \mathrm{H}, \mathrm{J}$ $=8.0 \mathrm{~Hz}, \mathrm{ArH}), 7.59\left(\mathrm{~A}\right.$ of $\left.\mathrm{AB}_{\mathrm{q}}, 4 \mathrm{H}, \mathrm{J}=9.0 \mathrm{~Hz}, \mathrm{ArH}\right), 7.61(\mathrm{~B}$ of $\mathrm{AB}, 4 \mathrm{H}$, $\mathrm{J}=9.0 \mathrm{~Hz}, \mathrm{ArH}) ;{ }^{13} \mathrm{C} \mathrm{NMR}$ and DEPT $\left(125 \mathrm{MHz}, \mathrm{CDCl}_{3}\right): \delta=148.0(\mathrm{C})$, 144.6 (C), 141.4 (C), 140.8 (C), 138.2 (C), 136.0 (C), 135.0 (C), 131.7 (C), $130.9(\mathrm{CH}), 129.3(\mathrm{CH}), 129.2$ (q, J = 32.4 Hz, C), 129.1 (C), 128.7 $(\mathrm{CH}), 127.9(\mathrm{CH}), 126.8(\mathrm{CH}), 126.5(\mathrm{CH}), 126.5(\mathrm{CH}), 126.4(\mathrm{CH}), 125.7$ $(\mathrm{CH}), 125.6(\mathrm{q}, \mathrm{J}=3.8 \mathrm{~Hz}, \mathrm{CH}), 124.6(\mathrm{CH}), 124.3(\mathrm{C}), 124.2(\mathrm{q}, \mathrm{J}=$ $\left.270.1 \mathrm{~Hz}, \mathrm{CF}_{3}\right), 122.8(\mathrm{CH}), 111.6(\mathrm{CH}), 50.8\left(\mathrm{CH}_{2}\right), 29.5\left(\mathrm{CH}_{2}\right), 20.4$ $\left(\mathrm{CH}_{2}\right), 14.0\left(\mathrm{CH}_{3}\right) ;{ }^{19} \mathrm{~F} \mathrm{NMR}\left(376 \mathrm{MHz}, \mathrm{CDCl}_{3}\right): \delta=-62.7$; IR (ATR): $v=$ $1607,1591,1319,1180,1161,1119,1107,1065,960,818 \mathrm{~cm}^{-1}$; MALDITOF (dithranol): $\mathrm{m} / \mathrm{z} 1269.7\left[\mathrm{M}+\mathrm{H}^{+}\right.$; ; elemental analysis calcd (\%) for $\mathrm{C}_{82} \mathrm{H}_{78} \mathrm{~F}_{6} \mathrm{~N}_{2} \mathrm{~S}_{2}$ : C 77.57, H 6,19, F 8,98, N 2,21; found: C 77.39, H 6.03, N 2.25 .

Compound 8. This compound was prepared from 3,3',5,5'-tetrabromo2,2'-bithiophene $(1 \mathrm{~g}, 2.07 \mathrm{mmol})$, 4-formylphenylboronic acid $(2.41 \mathrm{~g}$, $16.07 \mathrm{mmol}$ ), and tetrakis(triphenylphosphine)palladium(0) (116 mg, 0.10 $\mathrm{mmol}$ ) using the same procedure as described for 1 . The product was purified by washing with boiling EtOAc/EtOH (1:1 v/v). Yellow solid (800 $\mathrm{mg}, 67 \%)$. Further purification was achieved by crystallization from $\mathrm{CHCl}_{3} / \mathrm{MeOH}$. M.p. decomposes $>237^{\circ} \mathrm{C} ;{ }^{1} \mathrm{H}$ NMR $\left(500 \mathrm{MHz}, \mathrm{CDCl}_{3}\right): \delta$ $=7.12\left(\mathrm{~A}\right.$ of $\left.\mathrm{AB}_{\mathrm{q}}, 4 \mathrm{H}, \mathrm{J}=8.0 \mathrm{~Hz}, \mathrm{ArH}\right), 7.45(\mathrm{~s}, 2 \mathrm{H}, 2 \times \mathrm{CH}$ thiophene $)$, $7.62\left(\mathrm{~B}\right.$ of $\left.A B_{q}, 8 \mathrm{H}, J=8.0 \mathrm{~Hz}, \mathrm{ArH}\right), 7.80\left(\mathrm{~A}\right.$ of $\left.A B_{\mathrm{q}}, 4 \mathrm{H}, J=8.0 \mathrm{~Hz}, \mathrm{ArH}\right)$ $7.95\left(\mathrm{~B}\right.$ of $\left.\mathrm{AB}_{\mathrm{q}}, 8 \mathrm{H}, \mathrm{J}=8.0 \mathrm{~Hz}, \mathrm{ArH}\right), 9.95(\mathrm{~s}, 2 \mathrm{H}, 2 \times \mathrm{CH}=\mathrm{O}), 10.05$ (s, $2 \mathrm{H}, 2 \times \mathrm{CH}=\mathrm{O}) ;{ }^{13} \mathrm{C}$ NMR and DEPT $\left(125 \mathrm{MHz}, \mathrm{CDCl}_{3}\right): \delta=191.5$ $(\mathrm{CH}=\mathrm{O}), 191.2(\mathrm{CH}=\mathrm{O}), 143.9(\mathrm{C}), 141.3(\mathrm{C}), 141.3(\mathrm{C}), 138.7$ (C), 135.8 (C), $134.9(\mathrm{C}), 131.6(\mathrm{C}), 130.6(\mathrm{CH}), 129.6(\mathrm{CH}), 128.7(\mathrm{CH}), 127.0$ $(\mathrm{CH}), 126.0(\mathrm{CH})$; IR (ATR): $v=1693(\mathrm{C}=\mathrm{O}), 1595,1564,1209,1167$, $816 \mathrm{~cm}^{-1}$; MALDI-TOF (dithranol): $\mathrm{m} / z 582.8[\mathrm{M}]^{+}$; elemental analysis calcd (\%) for $\mathrm{C}_{36} \mathrm{H}_{22} \mathrm{O}_{4} \mathrm{~S}_{2}$ : C 74.21, H 3.81, S 11.01, found: C 74.30, H 3.82 .

Compound 9a. Solid $\mathrm{K}^{t} \mathrm{BuO}$ (555 mg, $4.95 \mathrm{mmol}$ ) was added in small portions to a solution of tetraldehyde $8(213 \mathrm{mg}, 0.37 \mathrm{mmol})$ and diethyl 4-(trifluoromethyl)benzylphosphonate $(490 \mathrm{mg}, 1.65 \mathrm{mmol})$ in dry THF $(15 \mathrm{~mL})$ under argon. The mixture was stirred at room temperature for 6 $\mathrm{h}$ and quenched with water. The THF was evaporated and the precipitate was filtered off and washed with $\mathrm{MeOH}$. Yellow solid (360 mg, 85\%). Further purification was achieved by crystallization from $\mathrm{CHCl}_{3} / \mathrm{MeOH}$. M.p. decomposes $>287^{\circ} \mathrm{C} ;{ }^{1} \mathrm{H}$ NMR $\left(500 \mathrm{MHz}, \mathrm{CDCl}_{3}\right.$ ): $\delta=7.08$ (A of $\left.A B_{q}, 2 \mathrm{H}, J=16.5 \mathrm{~Hz}, 2 \times \mathrm{CH}=\right), 7.15\left(\mathrm{~B}\right.$ of $\mathrm{AB}_{\mathrm{q}}, 2 \mathrm{H}, \mathrm{J}=16.5 \mathrm{~Hz}, 2 \times$ $\mathrm{CH}=), 7.15\left(\mathrm{~A}\right.$ of $\left.A B_{\mathrm{q}}, 4 \mathrm{H}, J=8.0 \mathrm{~Hz}, \mathrm{ArH}\right), 7.16\left(\mathrm{~A}\right.$ of $A B_{\mathrm{q}}, 2 \mathrm{H}, J=16.5$ $\mathrm{Hz}, 2 \times \mathrm{CH}=), 7.21$ ( $\mathrm{B}$ of $\left.\mathrm{AB}_{\mathrm{q}}, 2 \mathrm{H}, \mathrm{J}=16.5 \mathrm{~Hz}, 2 \times \mathrm{CH}=\right), 7.34$ ( $B$ of $A B_{\mathrm{q}}$, $4 \mathrm{H}, \mathrm{J}=8.0 \mathrm{~Hz}, \mathrm{ArH}), 7.39(\mathrm{~s}, 2 \mathrm{H}, 2 \times \mathrm{CH}$ thiophene), $7.57(\mathrm{~A}$ of $\mathrm{AB}, 4 \mathrm{H}$, $J=8.5 \mathrm{~Hz}, \mathrm{ArH}), 7.59\left(\mathrm{~A}\right.$ of $\left.\mathrm{AB}_{\mathrm{q}}, 4 \mathrm{H}, \mathrm{J}=8.5 \mathrm{~Hz}, \mathrm{ArH}\right), 7.62\left(\mathrm{~B}\right.$ of $A B_{q}, 4 \mathrm{H}$ $J=8.5 \mathrm{~Hz}, \mathrm{ArH}), 7.63(\mathrm{~s}, 8 \mathrm{H}, \mathrm{ArH}), 7.66\left(\mathrm{~B}\right.$ of $\left.\mathrm{AB}_{\mathrm{q}}, 4 \mathrm{H}, \mathrm{J}=8.0 \mathrm{~Hz}, \mathrm{ArH}\right)$; ${ }^{13} \mathrm{C}$ NMR and DEPT $\left(125 \mathrm{MHz}, \mathrm{CDCl}_{3}\right): \delta=144.1$ (C), 141.7 (C), 140.7 (C), 140.6 (C), 140.6 (C), 136.3 (C), 135.8 (C), 135.2 (C), 133.4 (C), $130.8(\mathrm{CH}), 130.4(\mathrm{CH}), 129.6(\mathrm{C}), 129.4$ (q, J = 32.6 Hz, C), 129.3 (q, J $=31.8 \mathrm{~Hz}, \mathrm{C}), 128.7(\mathrm{CH}), 127.4(\mathrm{CH}), 126.9(\mathrm{CH}), 126.6(\mathrm{CH}), 126.6$ $(\mathrm{CH}), 126.5(\mathrm{CH}), 125.8(\mathrm{CH}), 125.7(\mathrm{q}, \mathrm{J}=3.8 \mathrm{~Hz}, \mathrm{CH}), 125.7(\mathrm{q}, \mathrm{J}=3.8$ $\mathrm{Hz}, \mathrm{CH}), 125.3(\mathrm{CH}), 124.2\left(\mathrm{q}, \mathrm{J}=270.1 \mathrm{~Hz}, \mathrm{CF}_{3}\right) ;{ }^{19} \mathrm{~F}$ NMR $(376 \mathrm{MHz}$, $\mathrm{CDCl}_{3}$ ): $\delta=-62.49,-62.50 ;$ IR (ATR): $v=1612,1317,1159,1123,1107$, 1065, $833 \mathrm{~cm}^{-1}$; MALDI-TOF (dithranol): $\mathrm{m} / \mathrm{z} 1150.5 \mathrm{MM]}^{+}$; elemental analysis calcd (\%) for $\mathrm{C}_{68} \mathrm{H}_{42} \mathrm{~F}_{12} \mathrm{~S}_{2}$ : C 70.95, H 3.68, F 19.80, S 5.57; found: C 70.60, H, 3.46.

Compound $9 \mathrm{~b}$. This compound was prepared from $\mathrm{K}^{t} \mathrm{BuO}(650 \mathrm{mg}, 5.79$ $\mathrm{mmol})$, tetraldehyde $8(250 \mathrm{mg}, 0.43 \mathrm{mmol})$, and diethyl benzylphosphonate $(440 \mathrm{mg}, 1.93 \mathrm{mmol})$ using the same procedure as described for $9 \mathbf{a}$. Yellow solid $(300 \mathrm{mg}, 80 \%)$. Further purification was achieved by crystallization from EtOAc/MeOH. M.p. decomposes $>215$ ${ }^{\circ} \mathrm{C} ;{ }^{1} \mathrm{H}$ NMR $\left(500 \mathrm{MHz}, \mathrm{CDCl}_{3}\right): \delta=7.08(\mathrm{~s}, 4 \mathrm{H}, 4 \times \mathrm{CH}=), 7.12\left(\mathrm{~A}\right.$ of $\mathrm{AB}_{\mathrm{q}}$, $4 \mathrm{H}, \mathrm{J}=8.0 \mathrm{~Hz}, \mathrm{ArH}), 7.13\left(\mathrm{~A}\right.$ of $\left.\mathrm{AB}_{\mathrm{q}}, 4 \mathrm{H}, \mathrm{J}=16.0 \mathrm{~Hz}, 2 \times \mathrm{CH}=\right), 7.17(\mathrm{~B}$ of $\left.\mathrm{AB}_{\mathrm{q}}, 2 \mathrm{H}, \mathrm{J}=16.0 \mathrm{~Hz}, 2 \times \mathrm{CH}=\right), 7.26-7.30(\mathrm{~m}, 4 \mathrm{H}, \mathrm{ArH}), 7.31$ (B of $\left.\mathrm{AB}_{\mathrm{q}}, 4 \mathrm{H}, \mathrm{J}=8.0 \mathrm{~Hz}, \mathrm{ArH}\right), 7.36-7.40(\mathrm{~m}, 8 \mathrm{H}, \mathrm{ArH}), 7.37(\mathrm{~s}, 2 \mathrm{H}, 2 \times \mathrm{CH}$ thiophene), 7.51-7.55 (m, 8H, ArH), $7.55\left(\mathrm{~A}\right.$ of $\left.\mathrm{AB}_{\mathrm{q}}, 4 \mathrm{H}, \mathrm{J}=8.0 \mathrm{~Hz}, \mathrm{ArH}\right)$, $7.64\left(B\right.$ of $\left.A B_{q}, 4 \mathrm{H}, J=8.0 \mathrm{~Hz}, A r H\right) ;{ }^{13} \mathrm{C}$ NMR and DEPT $(125 \mathrm{MHz}$, $\mathrm{CDCl}_{3}$ ): $\delta=144.0$ (C), 141.7 (C), 137.3 (C), 137.2 (C), 136.9 (C), 135.8 (C), $135.2(\mathrm{C}), 132.9(\mathrm{C}), 129.3(\mathrm{C}), 128.9(\mathrm{CH}), 128.7(\mathrm{CH}), 128.7(\mathrm{CH})$ $128.6(\mathrm{CH}), 128.5(\mathrm{CH}), 128.4(\mathrm{CH}), 128.0(\mathrm{CH}), 127.8(\mathrm{CH}), 127.6(\mathrm{CH})$, $127.1(\mathrm{CH}), 126.6(\mathrm{CH}), 126.5(\mathrm{CH}), 126.3(\mathrm{CH}), 125.8(\mathrm{CH}), 125.2(\mathrm{CH})$ IR (ATR): $v=1593,1497,1072,964,814,752,688 \mathrm{~cm}^{-1}$; MALDI-TOF (dithranol): $\mathrm{m} / \mathrm{z} 878.3[\mathrm{M}]^{+}$; elemental analysis calcd (\%) for $\mathrm{C}_{64} \mathrm{H}_{46} \mathrm{~S}_{2}$ : C 87.43, H 5.27, S 7,29; found: C 87.34, H, 5.29.

Compound 9c. This compound was prepared from $\mathrm{K}^{\mathrm{t}} \mathrm{BuO}(258 \mathrm{mg}, 2.29$ $\mathrm{mmol})$, tetraldehyde $8(100 \mathrm{mg}, 0.17 \mathrm{mmol})$, and phosphonate $6(274 \mathrm{mg}$, $0.77 \mathrm{mmol}$ ) using the same procedure as described above for $\mathbf{9 a}$. Yellow solid (150 mg, 64\%). Further purification was achieved by crystallization from $\mathrm{DCM} / \mathrm{MeOH} .{ }^{1} \mathrm{H}$ NMR $\left(500 \mathrm{MHz}, \mathrm{CDCl}_{3}\right): \delta=1.36(\mathrm{~s}, 48 \mathrm{H}, 16 \times$ $\left.\mathrm{CH}_{3}\right), 7.06\left(\mathrm{~A}\right.$ of $\left.\mathrm{AB}_{\mathrm{q}}, 4 \mathrm{H}, \mathrm{J}=16.5 \mathrm{~Hz}, 2 \times \mathrm{CH}=\right), 7.09(\mathrm{~A}$ of $\mathrm{AB}, 4 \mathrm{H}, \mathrm{J}=$ $8.0 \mathrm{~Hz}, \mathrm{ArH}), 7.13\left(\mathrm{~B}\right.$ of $\left.A B_{\mathrm{q}}, 4 \mathrm{H}, J=16.5 \mathrm{~Hz}, 2 \times \mathrm{CH}=\right), 7.14\left(\mathrm{~A}\right.$ of $A B_{\mathrm{q}}$ $4 \mathrm{H}, J=16.5 \mathrm{~Hz}, 2 \times \mathrm{CH}=), 7.19\left(\mathrm{~B}\right.$ of $\left.\mathrm{AB}_{\mathrm{q}}, 2 \mathrm{H}, J=17.0 \mathrm{~Hz}, 2 \times \mathrm{CH}=\right)$, 7.29 ( $\mathrm{B}$ of $\left.\mathrm{AB}_{\mathrm{q}}, 4 \mathrm{H}, \mathrm{J}=8.0 \mathrm{~Hz}, \mathrm{ArH}\right), 7.35(\mathrm{~s}, 2 \mathrm{H}, 2 \times \mathrm{CH}$ thiophene), 7.50 ( $A$ of $\left.A B_{q}, 4 \mathrm{H}, J=8.0 \mathrm{~Hz}, A r H\right), 7.53\left(A\right.$ of $A B_{q}, 4 \mathrm{H}, J=8.0 \mathrm{~Hz}, A r H$ ), $7.54\left(A\right.$ of $\left.A B_{q}, 4 \mathrm{H}, J=8.0 \mathrm{~Hz}, \operatorname{ArH}\right), 7.62\left(B\right.$ of $\left.A B_{q}, 4 \mathrm{H}, J=8.0 \mathrm{~Hz}, A r H\right)$, $7.80\left(B\right.$ of $\left.A B_{q}, 4 \mathrm{H}, J=7.5 \mathrm{~Hz}, A r H\right), 7.81\left(B\right.$ of $A B_{q}, 4 H, J=8.0 \mathrm{~Hz}$, $\mathrm{ArH}) ;{ }^{13} \mathrm{C}$ NMR and DEPT (125 MHz, $\left.\mathrm{CDCl}_{3}\right): \delta=144.0$ (C), 141.7 (C), 140.0 (C), 139.9 (C), 136.8 (C), 135.7 (C), 135.4 (C), $135.2(\mathrm{CH}), 134.7$ (C), $133.1(\mathrm{C}), 129.4(\mathrm{C}), 129.3(\mathrm{CH}), 128.9(\mathrm{CH}), 128.8(\mathrm{CH}), 128.6$ $(\mathrm{CH}), 128.4(\mathrm{CH}), 128.1(\mathrm{C}), 127.2(\mathrm{CH}), 126.4(\mathrm{CH}), 125.8(\mathrm{CH}), 125.8$ $(\mathrm{CH}), 125.8(\mathrm{CH}), 125.2(\mathrm{CH}), 83.8(\mathrm{C}), 83.8(\mathrm{C}), 24.9\left(\mathrm{CH}_{3}\right)$; elemental analysis calcd (\%) for $\mathrm{C}_{88} \mathrm{H}_{90} \mathrm{~B}_{4} \mathrm{O}_{8} \mathrm{~S}_{2}: \mathrm{C} 76.42, \mathrm{H} 6.56$, B 3.13, S 4.64; found: C 76.20, H, 6.41.

Compound $9 \mathrm{~d}$. This compound was prepared from $\mathrm{K}^{t} \mathrm{BuO}(202 \mathrm{mg}, 1.80$ $\mathrm{mmol})$, dialdehyde $4 \mathrm{~b}(200 \mathrm{mg}, 0.24 \mathrm{mmol})$, and diethyl 4-tertbutylbenzylphosphonate $(171 \mathrm{mg}, 0.60 \mathrm{mmol})$ using the same procedure as described for $9 \mathbf{a}$. The crude product was purified by column chromatography $\left(\mathrm{SiO}_{2}\right.$, hexanes/EtOAc, 8:2) and washed with boiling EtOH. Orange solid (130 mg, 49\%). M.p. decomposes > $206{ }^{\circ} \mathrm{C} ;{ }^{1} \mathrm{H}$ NMR $\left(500 \mathrm{MHz}, \mathrm{CDCl}_{3}\right): \delta=1.34\left(\mathrm{~s}, 36 \mathrm{H}, 12 \times \mathrm{CH}_{3}\right), 7.00\left(\mathrm{~A}^{\circ} \mathrm{AB}_{\mathrm{q}}, 2 \mathrm{H}, \mathrm{J}=\right.$ 16.0 Hz, $2 \times \mathrm{CH}=), 7.05\left(\mathrm{~B}\right.$ of $\left.\mathrm{AB}_{\mathrm{q}}, 2 \mathrm{H}, \mathrm{J}=16.5 \mathrm{~Hz}, 2 \times \mathrm{CH}=\right), 7.07$ ( $\mathrm{A}$ of $\left.\mathrm{AB}_{\mathrm{q}}, 2 \mathrm{H}, \mathrm{J}=16.0 \mathrm{~Hz}, 2 \times \mathrm{CH}=\right), 7.09\left(\mathrm{~A}\right.$ of $\left.\mathrm{AB}_{\mathrm{q}}, 4 \mathrm{H}, \mathrm{J}=8.5 \mathrm{~Hz}, \mathrm{ArH}\right)$ $7.13\left(B\right.$ of $\left.A B_{q}, 2 H, J=16.5 \mathrm{~Hz}, 2 \times C H=\right), 7.27\left(B\right.$ of $A B_{q}, 4 H, J=8.5 \mathrm{~Hz}$, $\mathrm{ArH}), 7.33\left(\mathrm{~s}, 2 \mathrm{H}, 2 \times \mathrm{CH}\right.$ thiophene), $7.38\left(\mathrm{~A}\right.$ of $\mathrm{AB}_{\mathrm{q}}, 4 \mathrm{H}, J=8.0 \mathrm{~Hz}$, $\mathrm{ArH}), 7.39\left(\mathrm{~A}\right.$ of $\left.A B_{\mathrm{q}}, 4 \mathrm{H}, J=8.5 \mathrm{~Hz}, \mathrm{ArH}\right), 7.44\left(\mathrm{~B}_{\mathrm{A}} \mathrm{AB}, 4 \mathrm{H}, J=8.5 \mathrm{~Hz}\right.$, $\mathrm{ArH}), 7.46\left(\mathrm{~B}\right.$ of $\left.A B_{q}, 4 \mathrm{H}, J=8.0 \mathrm{~Hz}, \mathrm{ArH}\right), 7.51\left(\mathrm{~A}\right.$ of $A B_{q}, 4 \mathrm{H}, J=8.5 \mathrm{~Hz}$, $\mathrm{ArH}), 7.60\left(\mathrm{~B}\right.$ of $\left.\mathrm{AB}_{\mathrm{q}}, 4 \mathrm{H}, \mathrm{J}=8.5 \mathrm{~Hz}, \mathrm{ArH}\right) ;{ }^{13} \mathrm{C}$ NMR and DEPT (125 $\left.\mathrm{MHz}, \mathrm{CDCl}_{3}\right): \delta=150.9$ (C), 150.7 (C), 144.0 (C), 141.7 (C), 137.1 (C), 136.0 (C), 135.0 (C), 134.6 (C), 134.4 (C), 132.7 (C), 129.3 (C), 128.7 $(\mathrm{CH}), 128.6(\mathrm{CH}), 128.2(\mathrm{CH}), 127.6(\mathrm{CH}), 127.2(\mathrm{CH}), 126.9(\mathrm{CH}), 126.3$ $(\mathrm{CH}), 126.2(\mathrm{CH}), 126.2(\mathrm{CH}), 125.7(\mathrm{CH}), 125.6(\mathrm{CH}), 125.6(\mathrm{CH}), 125.1$ $(\mathrm{CH}), 34.6(\mathrm{C}), 34.6(\mathrm{C}), 31.3\left(\mathrm{CH}_{3}\right)$; IR (ATR): $v=1514,1362,1107,962$, $826 \mathrm{~cm}^{-1}$; MALDI-TOF (dithranol): $\mathrm{m} / \mathrm{z} 1102.3\left[\mathrm{M}^{+}\right.$; elemental analysis calcd (\%) for $\mathrm{C}_{80} \mathrm{H}_{78} \mathrm{~S}_{2}$ : C 87.07, H 7.12, S 5.81; found: C 87.15, H, 7.14.

\section{Acknowledgements}

This research was funded by the Ministerio de Economía y Competitividad (Spain)/FEDER (EU) (project BFU2011-30161C02-02) and the Junta de Comunidades de Castilla-La Mancha (JCCM)/FEDER (EU) (project PEII-2014-005-A). A.G.-R. thanks JCCM for a doctoral fellowship. 
Keywords: Swivel cruciforms • Sulfur heterocycles • Stilbenes • Conjugation • Donor-acceptor systems

[1] a) Special Issue on Conducting Polymers for Carbon Electronics, Chem Soc. Rev. 2010, 39, 2337-2732; b) A. C. Grimsdale, K. L. Chan, R. E. Martin, P. G. Jokisz, A. B. Holmes, Chem. Rev. 2009, 109, 897-1091 C) S. R. Forrest, M. E. Thompson, Chem. Rev. 2007, 107, 923-925; d) Organic Light Emitting Devices: Synthesis, Properties and Applications (Eds.: K. Müllen, U. Sherf), Wiley-VCH, Weinheim, 2006; e) H. Meier Angew. Chem. Int. Ed. 2005, 44, 2482-2506; f) Special Issue on Organic Electronics, Chem. Mater. 2004, 16, 4381-4846; g) R. H. Friend, R. W. Gymer, A. B. Holmes, J. H. Burroughes, R. N. Marks, C. Taliani, D. D. C. Bradley, D. A. Dos Santos, J. L. Brédas, M. Lögdlund, W. R. Salaneck, Nature 1999, 397, 121-128; h) H. Sirringhaus, N Tessler, R. H. Friend, Science 1998, 280, 1741-1744; i) Conjugated Polymers and Related Materials. The Interconnection of Chemical and Electronic Structure (Eds.: W. R. Salaneck, I. Lundström, B. G. Rånby), Oxford University Press, Oxford, 1993.

[2] A. Mangalum, R. C. Smith, Dalton Trans. 2010, 39, 5145-5151.

[3] a) K. Osowska, O. S. Miljanić, Chem. Commun. 2010, 46, 4276-4278 b) W. W. Gerhardt, A. J. Zucchero, C. R. South, U. H. F. Bunz, M. Weck, Chem.-Eur. J. 2007, 13, 4467-4474; c) W. W. Gerhardt, A. J. Zucchero, J. N. Wilson, C. R. South, U. H. F. Bunz, M. Weck, Chem. Commun. 2006, 2141-2143; d) G. M. Florio, J. E. Klare, M. O Pasamba, T. L. Werblowsky, M. Hyers, B. J. Berne, M. S. Hybertsen, C. Nuckolls, G. W. Flynn, Langmuir 2006, 22, 10003-10008; e) J. E. Klare, G. S. Tulevski, C. Nuckolls, Langmuir 2004, 20, 10068-10072.

[4] a) S. Grunder, R. Huber, W. Songmei, C. Schönenberg, M. Calame, M. Mayor, Eur. J. Org. Chem. 2010, 833-845; b) S. Grunder, R. Huber, V. Horhoiu, M. T. González, C. Schönenberg, M. Calame, M. Mayor, J. Org. Chem. 2007, 72, 8337-8344.

[5] a) H. Luo, S. Chen, Z. Liu, C. Zhang, Z. Cai, X. Chen, G. Zhang, Y. Zhao, S. Decurtins, S.-X. Liu, D. Zhang, Adv. Funct. Mater. 2014, 24 4250-4258; b) X. Zhang, X. Jiang, J. Luo, C. Chi, H. Chen, J. Wu, Chem.-Eur. J. 2010, 16, 464-468; c) C. Wang, Y. Liu, Z. Ji, E. Wang, R. Li, H. Jiang, Q. Tang, H. Li, W. Hu, Chem. Mater. 2009, 21, 28402845.

[6] a) J. Sun, H. Lai, H. Zhong, Q. Fang, Thin Solid Films 2011, 519, 77727778; b) S. Liu, F. He, H. Wang, H. Xu, C. Wang, F. Li, Y. Ma, J. Mater. Chem. 2008, 18, 4802-4807; c) J. Sun, J.-G. Cheng, W.-Q. Zhu, S.-J. Ren, H.-L. Zhong, D.-L. Zeng, J.-P. Du, E.-J. Xu, Y.-C. Liu, Q. Fang, J. Polym. Sci., Part A: Polym. Chem. 2008, 46, 5616-5625.

[7] a) M. J. Cho, S. S. Park, Y. S. Yang, J. H. Kim, D. H. Choi, Synth. Met. 2010, 160, 1754-1760; b) W. Cui, Y. Fu, Y. Qu, H. Tian, J. Zhang, Z. Xie, Y. Geng, F. Wang, Chem.-Asian J. 2010, 5, 932-940; c) Y.-G. Kim H. Christian-Pandya, N. Ananthakrishnan, Z. I. Niazimbetova, B. C. Thompson, M. E. Galvin, J. R. Reynolds, Sol. Energy Mater. Sol. Cells 2008, 92, 307-312.

[8] a) M. A. Saeed, H. T. M. Le, O. Š. Miljanić, Acc. Chem. Res. 2014, 47, 2074-2083; b) J. Tolosa, K. M. Solntsev, L. M. Tolbert, U. H. F. Bunz, J. Org. Chem. 2010, 75, 523-534; c) J. Tolosa, U. H. F. Bunz, Chem.Asian J. 2009, 4, 270-276, d) J. Tolosa, A. J. Zucchero, U. H. F. Bunz, J. Am. Chem. Soc. 2008, 130, 6498-6506; e) P. L. McGrier, K. M. Solntsev, S. Miao, L. M. Tolbert, O. R. Miranda, V. M. Rotello, U. H. F. Bunz, Chem.-Eur. J. 2008, 14, 4503-4510; f) S. M. Brombosz, A. J. Zucchero, R. L. Phillips, D. Vazquez, A. Wilson, U. H. F. Bunz, Org. Lett. 2007, 22, 4519-4522, g) M. Hauck, J. Schönhaber, A. J. Zucchero,
K. I. Hardcastle, T. J. J. Müller, U. H. F. Bunz, J. Org. Chem. 2007, 72, 6714-6725; h) P. L. McGrier, K. M. Solntsev, J. Schönhaber, S. M Brombosz, L. M. Tolbert, U. H. F. Bunz, Chem. Commun. 2007, 21272129; i) A. J. Zucchero, J. N. Wilson, U. H. F. Bunz, J. Am. Chem. Soc 2006, 128, 11872-11881; j) J. N. Wilson, U. H. F. Bunz, J. Am. Chem. Soc. 2005, 127, 4124-4125.

[9] a) T. Krishnamoorthy, F. Kunwu, P. P. Boix, H. Li, T. M. Koh, W. L. Leong, S. Powar, A. Grimsdale, M. Grätzel, N. Mathews, S. G Mhaisalkar, J. Mater. Chem. A 2014, 2, 6305-6309; b) H. Wang, T. Fukumatsu, Y. Liu, W. Hu, S. Seki, X. Zhan, J. Mater. Chem. C 2013, 1 414-417; c) D. Ni, B. Zhao, T. Shi, S. Ma, G. Tu, H. Wu, ACS Macro Lett. 2013, 2, 621-624; d) S. Xue, S. Liu, F. He, L. Yao, C. Gu, H. Xu, Z. Xie, H. Wu, Y. Ma, Chem. Commun. 2013, 49, 5730-5732; e) J.-M. Koenen, A. Bilge, S. Allard, R. Alle, K. Meerholz, U. Scherf, Org. Lett. 2009, 11, 2149-2152; f) A. Zen, P. Pingel, F. Jaiser, D. Neher, J. Grenzer, W. Zhuang, J. P. Rabe, A. Bilge, F. Gallbrecht, B. S. Nehls, T. Farrell, U. Scherf, R. D. Abellon, F. C. Grozema, L. D. A. Siebbeles, Chem. Mater. 2007, 19, 1267-1276; g) S. Karpe, A. Cravino, P. Frère, M. Allain, G. Mabon, J. Roncali, Adv. Funct. Mater. 2007, 17, 11631171; h) A. Wakamiya, K. Mori, S. Yamaguchi, Angew. Chem. Int. Ed. 2007, 46, 4273-4276; i) A. Bilge, A. Zen, M. Forster, H. Li, F. Gallbrecht, B. S. Nehls, T. Farrell, D. Neher, U. Scherf, J. Mater. Chem. 2006, 16, 3177-3182; j) A. Zen, A. Bilge, F. Galbrecht, R. Alle, K. Meerholz, J. Grenzer, D. Neher, U. Scherf, T. Farrell, J. Am. Chem. Soc. 2006, 128, 3914-3915.

[10] C. Romero-Nieto, S. Merino, J. Rodríguez-López, T. Baumgartner, Chem.-Eur. J. 2009, 15, 4135-4145.

[11] M. Scheuble, M. Goll, S. Ludwigs, Macromol. Rapid Commun. 2014 DOI: $10.1002 / \mathrm{marc} .201400525$

[12] See for example: A. Pérez, J. L. Serrano, T. Sierra, A. Ballesteros, D. de Saá, R. Termine, U. K. Pandey, A. Golemme, New J. Chem. 2012, 36, 830-842.

[13] D. F. Eaton, Pure Appl. Chem. 1988, 60, 1107-1114.

[14] a) P. C. Hariharan, J. A. Pople, Mol. Phys. 2006, 27, 209-214; b) P. C. Hariharan, J. A. Pople, Theoret. Chim. Acta 1973, 28, 213-222; c) W. J. Hehre, R. Ditchfield, J. A. Pople, J. Chem. Phys. 1972, 56, 2257-2261; d) R. Ditchfield, W. J. Hehre, J. A. Pople, J. Chem. Phys. 1971, 54, 724-728.

[15] a) A. D. Becke, J. Chem. Phys. 1993, 98, 5648-5652; b) C. T. Lee, W. T. Yang, R. G. Parr, Phys. Rev. B 1988, 37, 785-789.

[16] M. J. Frisch, G. W. Trucks, H. B. Schlegel, G. E. Scuseria, M. A. Robb J. R. Cheeseman, G. Scalmani, V. Barone, B. Mennucci, G. A. Petersson, H. Nakatsuji, M. Caricato, X. Li, H. P. Hratchian, A. F. Izmaylov, J. Bloino, G. Zheng, J. L. Sonnenberg, M. Hada, M. Ehara, K. Toyota, R. Fukuda, J. Hasegawa, M. Ishida, T. Nakajima, Y. Honda, O Kitao, H. Nakai, T. Vreven, J. A. Montgomery, Jr., J. E. Peralta, F. Ogliaro, M. J. Bearpark, J. J. Heyd, E. N. Brothers, K. N. Kudin, V. N. Staroverov, R. Kobayashi, J. Normand, K. Raghavachari, A. P. Rendell, J. C. Burant, S. S. Iyengar, J. Tomasi, M. Cossi, N. Rega, N. J. Millam, M. Klene, J. E. Knox, J. B. Cross, V. Bakken, C. Adamo, J. Jaramillo, R. Gomperts, R. E. Stratmann, O. Yazyev, A. J. Austin, R. Cammi, C. Pomelli, J. W. Ochterski, R. L. Martin, K. Morokuma, V. G. Zakrzewski, G. A. Voth, P. Salvador, J. J. Dannenberg, S. Dapprich, A. D. Daniels, Ö. Farkas, J. B. Foresman, J. V. Ortiz, J. Cioslowski, D. J. Fox in Gaussian 09, Revision B.01; Gaussian Inc., Wallingford, CT, 2009. 
Entry for the Table of Contents (Please choose one layout)

Layout 1:

\section{FULL PAPER}

Bithiophene-based cruciforms with different stilbenoid arms at the 3,3'and 5,5'-positions have been synthesized. The steric hindrance between the stilbene arms at the 3,3'positions makes the molecules adopt a twisted geometry that results in weaker intermolecular interactions and better solubility in common organic solvents. The electrochemical results demonstrate that these systems can support stable radical cations and show their potential as hole-transporting materials.

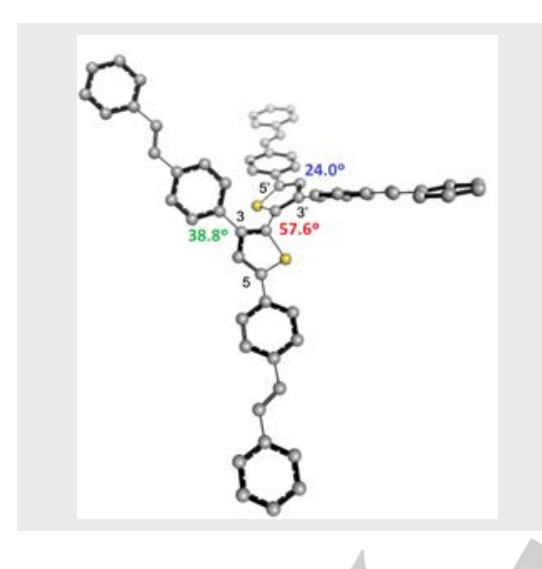

Amaranda García-Rodríguez, Antonio M. Rodríguez, Pilar Prieto, Raquel Andreu, Sonia Merino, * Julián Rodríguez-López*

Page No. - Page No.

Swivel-Cruciform Stilbenes Based on Bithiophene

Layout 2:

\section{FULL PAPER}

((Insert TOC Graphic here; max. width: $11.5 \mathrm{~cm}$; max. height: $2.5 \mathrm{~cm}$ ))
Author(s), Corresponding Author(s)*

Page No. - Page No.

Title

Text for Table of Contents 